\title{
Estimation of Vegetation Structure Parameters from SMAP Radar Intensity Observations
}

\author{
Thomas Jagdhuber, Senior Member, IEEE, Carsten Montzka, Member, IEEE, Carlos Lopez- \\ Martinez, Senior Member, IEEE, Martin J. Baur, Student Member, IEEE, Moritz Link, \\ María Piles, Senior Member, IEEE, Narendra N. Das, François Jonard
}

\begin{abstract}
In this research study we present a multipolarimetric estimation approach for two model-based vegetation structure parameters (shape $A_{P} \&$ orientation distribution $\psi$ of main canopy elements). The approach is based on a reduced observation set of three incoherent (no phase information) polarimetric backscatter intensities $\left(\left|S_{H H}\right|^{2},\left|S_{H V}\right|^{2},\left|S_{V V}\right|^{2}\right)$ combined with a two-parameter $\left(A_{P} \& \psi\right)$ discrete scatterer model of vegetation. The objective is to understand if this confined set of observations contains enough information to estimate the two vegetation structure parameters from $L$-band radar signals. In order to disentangle soil and vegetation scattering influences on these signals and ultimately perform a vegetation-only retrieval of vegetation shape $A_{P}$ and orientation distribution $\psi$, we use the sub-pixel spatial heterogeneity expressed by the covariation of co- and cross-polarized backscatter $\Gamma_{P P-P Q}$ of the neighboring cells and assume it is indicative for the amount of a vegetation-only co-to-crosspolarized backscatter ratio $\mu_{P P-P Q}$. The ratio-based retrieval approach enables a relative (no absolute backscatter) estimation of the vegetation structure parameters which is more robust compared to retrievals with absolute terms.

The application of the developed algorithm on global L-band SMAP radar data acquired from April to July 2015 indicates the potential and limitations of estimating these two parameters when no fully polarimetric data is available. A focus study on six different regions of interest, spanning land cover from barren
\end{abstract}

Manuscript received: $20^{\text {th }}$ of March 2020.

This work was supported with the MIT-Germany Seed Fund "Global Water Cycle and Environmental Monitoring using Active and Passive Satellite-based Microwave Instruments" and with the MIT-Belgium UCL Seed Fund "Early Detection of Plant Water Stress Using Remote Sensing". C. Montzka is supported by the European Commission Horizon 2020 Program that funded the ERA-PLANET/GEOEssential (Grant Agreement no. 689443) project. M. Piles received partial funding through project RTI2018-096765-A-100 (MCIU/AEI/FEDER, UE)

T. Jagdhuber, Martin J. Baur and Moritz Link are affiliated with the Microwaves and Radar Institute, German Aerospace Center, 82234 Wessling, Germany (e-mails: thomas.jagdhuber@dlr.de, moritzlink0@gmail.com).

C. Montzka and F. Jonard are with the Institute of Bio- and Geoscience, Research Center Jülich, 52428 Jülich, Germany (e-mails: c.montzka@fzjuelich.de, f.jonard@fz-juelich.de).

F. Jonard is also with the Earth and Life Institute, Université catholique de Louvain, 1348 Louvain-la-Neuve, Belgium (email: francois.jonard @uclouvain.be).

Carlos Lopez-Martinez is with the Department of Signal Theory and Communications, Universitat Polytècnica de Catalunya, 08034 Barcelona, Spain (e-mail: carlos.lopez@tsc.upc.edu).

Martin J. Baur is with the Center of Ecology and Environmental Research, University of Bayreuth, 95447 Bayreuth, Germany (email: Martin.Baur@unibayreuth.de).

María Piles is with the Image Processing Lab, University of Valencia, 46980 Paterna, Spain (e-mail: maria.piles@uv.es).

Narendra N. Das is with the Jet Propulsion Laboratory, California Institute of Technology, 91109 Pasadena, USA (email: Narendra.N.Das@jpl.nasa.gov). land to tropical rainforest, shows a steady increase of orientation distribution towards randomly oriented volumes and a continuous decrease in shape arriving at dipoles for tropical vegetation. A comparison with independent datasets of vegetation height and above ground biomass confirms this consistent and meaningful retrieval of $A_{P}$ and $\psi$. The retrieved shapes and orientation distributions represent main vegetation elements matching literature results from model-based decompositions of fully polarimetric L-band data at the SMAP spatial resolution. Based on our findings, $A_{P}$ and $\psi$ can be directly applied for parameterizing the vegetation scattering component of modelbased polarimetric decompositions. This should facilitate decomposition into ground and vegetation scattering components and improve retrieval of soil parameters (moisture \& roughness) under vegetation.

Index Terms - radar, polarimetry, scattering, vegetation structure, vegetation model, discrete scatterer, SMAP

\section{INTRODUCTION}

$\mathrm{T}$ $\mathrm{HE}$ advantage of radar signals at L-band (typically at $\sim 1.26 \mathrm{GHz}$ ) is that in most cases major parts of the vegetation volume are penetrated and not just the top of the canopy as occurs at shorter wavelengths (e.g. C- and X-band) [1]. The backscattering signal is a mixture of soil and vegetation contributions, which need to be disentangled according to the focus of the study. The vegetation component contains important information for the characterization of plant biophysical parameters, such as shape, size, orientation and distribution of plant elements, water content, plant height, leaf area index, aboveground biomass, or plant stress [2], [3], [4], [5]. Both diurnal [6] and seasonal [7] time scales can be observed, making radar an important tool to assess process feedbacks in the soil-vegetation-atmosphere system [8].

Main vegetation scattering methods consider vegetation as discrete dielectric scattering objects randomly located in space (e.g., [9]). First simple semi-empirical models simulated vegetation as a water cloud whose droplets are held in place by the vegetative matter [10]. In these water cloud models, the backscattering coefficient was treated as a function of the target parameters soil moisture, vegetation water content and plant height, where scattering and attenuation cross-section contributions of the signal path through the canopy were implemented. The canopy is regarded as a uniform layer of some specified height containing a random distribution of discrete scatterers, and only single scattering is accounted for [11]. Later, further parameters such as leaf size [12] and leaf area index [13] were introduced. Kweon and $\mathrm{Oh}$ in [14] 
modified the water cloud model by implementing the average and standard deviation of leaf angle distribution for improved estimation of the backscattering coefficients with the angular effect of scattering particles in a vegetation canopy. However, the uniform random distribution of scatterers introduces inconsistencies during the vegetation parameter retrieval process mainly over forests. More advanced canopy scattering models such as the Michigan Microwave Canopy Scattering Model (MIMICS) were developed to better represent the transmission of energy through the multi-layer scattering medium. MIMICS divides the canopy into three components, i.e. the crown, the trunk and the underlying ground region [15]. Here, probability density functions (PDFs) of size, diameter and orientation of these three canopy components are implemented. Typically, radar signals from vegetated surfaces comprise contributions of direct backscatter from the vegetation itself, backscatter from the soil that is attenuated by the canopy and backscatter due to interactions between the vegetation and the underlying soil [16]. Therefore, polarimetric decomposition methods use, for instance, simple physics-based scattering models to separate total scattering from a target into its elementary scattering contributions [17]. Volume scattering from the canopy, surface scattering from a rough ground and double-bounce scattering from ground and stem are separated within the polarimetric covariance or coherency matrix. Cloude and Pottier in [18] described target scattering by eigenvectors to trace the scattering mechanism and eigenvalues to characterize the intensity of each mechanism.

Scattering from vegetation canopies is a result of multiple scattering within the canopy, and between the canopy and the ground [19]. Where early vegetation scattering studies focused on co-polarized backscatter (HH and VV; e.g. [12]), the inclusion of cross-polarized backscatter ( $\mathrm{HV}$ and $\mathrm{VH}$ ), indicative of these multiple scattering events, provided improved retrieval of vegetation information such as leaf area index and biomass in many studies (e.g., [20], [21]). Similar improvements were facilitated by implementing complex scattering mechanisms in the parameter retrieval. In addition to polarimetry, vegetation changes also impact the phase diversity [22], a research field currently not fully explored. Moreover, also synthetic aperture radar (SAR) tomography evolved during the last two decades towards three dimensional (3D) scattering response analysis for vegetation characterization or reconstruction by multi-baseline interferometric SAR [23], [24], [25], [26].

The problem of using advanced and more complex models to forward calculate radar backscatter is that a large number of parameters is needed. This data collection requirement may be attainable during intensive field campaigns, but it is too time consuming and expensive to be performed globally and regularly and for all types of vegetation covers [19]. Model inversion approaches to estimate those parameters typically make use of multi-angular [27], multi-frequency [28], multitemporal [29] observations, interferometry [30], [31] or a combination of multiple setups [32].

In this study we develop a multi-polarimetric estimation approach for vegetation structure parameters which is based on a reduced observation set of three incoherent (no phase information) polarimetric intensities, combined with a discrete scatterer model of vegetation. The research objective is to understand if this confined set of information can be sufficient to estimate vegetation structure parameters (shape and orientation distribution of main canopy elements). Hence, the requirement for this study is to develop a model-based approach to estimate two vegetation structure parameters with the limited observation set of three backscatter intensities (HH, VV, HV). The developed approach is applied to NASA's Soil Moisture Active Passive (SMAP) radar intensity observations, which were recorded in the period from April $13^{\text {th }} 2015$ to July $7^{\text {th }} 2015$.

First, we describe the characteristics of the SMAP data and its pre-processing (Section II.). Second, the scattering mechanisms occurring in the vegetation canopy are explained (Section III. A.) and a sensitivity experiment to identify the importance of the two main vegetation parameters (shape $A_{P}$ and orientation distribution $\psi$ of main canopy elements) for the prediction of volume scattering is introduced (Section III. B.) and conducted (Section IV.A). An inverse retrieval approach for the mentioned vegetation parameters is developed in Sections III. C \& III. D. An application of the proposed approach to SMAP active radar data is presented in Section IV.B and compared against independent datasets of vegetation height and biomass in IV.C. Results are discussed in Section V and conclusions are drawn in Section VI together with a short outlook to future research.

\section{DATA}

The Soil Moisture Active Passive (SMAP) mission of National Aeronautics and Space Agency (NASA) was launched in 2015 to acquire active and passive microwave measurements and produce global maps of soil moisture and freeze/thaw-states in a three-days-cycle [41]. It was designed to record radiometer and SAR observations with a shared Lband (1.26 GHz for $\mathrm{H}$ and $1.29 \mathrm{GHz}$ for $\mathrm{V}$ ) horn antenna and a spinning mesh reflector [41], [47]. The data are acquired with a fixed incidence angle at $40^{\circ}$ (off nadir) using a conical scan across a swath of $1000 \mathrm{~km}$ and at fixed local time (6 a.m.) [41]. The SAR instrument acquires backscatter (intensity, no phase) in $\mathrm{HH}$ and VV co-polarization and in one crosspolarization ( $\mathrm{HV}$ or $\mathrm{VH})$ with a relative radiometric accuracy of $0.5 \mathrm{~dB}$ and a noise equivalent sigma zero (NESZ) of $-30 \mathrm{~dB}$ [42], [48], [49]. Due to the malfunction of the SAR instrument on $7^{\text {th }}$ of July 2015 , only an 11-week period of global acquisitions -from April $13^{\text {th }}$ until July $7^{\text {th }}$ 2015- can be utilized for our analyses. Given this short acquisition period, we discard analyses of temporal dynamics due to limited statistical representativeness.

SMAP space-borne multi-polarimetric SAR intensity observations (more specifically $\left|S_{H H}\right|^{2},\left|S_{V V}\right|^{2}$ and $\left|S_{H V}\right|^{2}$, see section III) are available on a global basis for the measurement period [41], [42]. The SAR data is processed on a nominal spatial resolution of $3 \mathrm{~km}$ (70\% outer regions of swath) [47] [50], but resampled to $9 \mathrm{~km}$ posting [46]. A mask for deserts, water bodies and urban areas was applied to filter out areas 
where the retrieval algorithm does not apply [43], [44]. Table I summarizes the SMAP radar data specifications. Further details are given in [42].

TABLE I: SPECIFICATIONS OF APPLIED SMAP RADAR DATA FOR INPUT INTO THE VEGETATION PARAMETER ESTIMATION ALGORITHM [42], [47] - [49].

\begin{tabular}{cc} 
Specification & Global SMAP Level 2 radar data \\
\hline Frequency & L-band \\
Polarizations & $\mathrm{HH}, \mathrm{VV}, \mathrm{HV}$ (intensity, no phase) \\
Acquisition period & April 13, 2015 to July 7, 2015 \\
Temporal revisit & $2-3$ days (depending on latitude) \\
Incidence angle & $40^{\circ}$ (conical scan) \\
Spatial resolution & $9 \mathrm{~km}$ (re-gridded from 3 km) \\
Radiometric resolution & $0.5 \mathrm{~dB}$ \\
NESZ & $-30 \mathrm{~dB}$ \\
Incidence angle & $40^{\circ}$ \\
\hline
\end{tabular}

The 2005 MODIS MCD12Q1 International GeosphereBiosphere Program (IGBP) collection 5 land cover product is used in Section IV to interpret the estimation results on modelbased vegetation structure parameters $\left(A_{P}, \psi\right)$ in terms of 17 land cover classes. It is a global product with $500 \mathrm{~m}$ spatial resolution and freely available from the U.S. Land Processed Distributed Active Archive Center (www.lpdaac.usgs.gov). Independent data sets of vegetation height \& above ground biomass were selected for later comparison of the retrieved vegetation structure parameters (in Section IV.C). The vegetation height data is based on LiDAR measurements of the Geoscience Laser Altimeter System (GLAS) sensor on the ICESat platform combined with climatology and remote sensing of the optical bands [51]. The above ground biomass estimates is derived from the ESA GlobBiomass project for the year 2010 by combining space-borne SAR, LiDAR and optical observations together with auxiliary datasets from forest inventories, climatological variables and ecosystems classifications [52]. Both datasets were re-gridded to match the SMAP radar data (9 $\mathrm{km}$ grid).

\section{INTENSITY-BASED VEGETATION PARAMETER ESTIMATION USING A DISCRETE SCATTERER MODEL}

Predictions for vegetation volume backscattering are often conducted by discrete vegetation scattering models [5], [7]. Here, discrete scattering elements of the vegetation canopy, also called inclusions, are brought into a homogenous background medium, mostly air in case of naturally vegetated environments [33], [34], [35]. This allows backscatter simulations from a (homogenously) filled layer of discrete inclusions on top of a single-scattering soil surface, like in [36].
The following subsections (A to D) exemplify how radar intensity data (no phase information) can be used to retrieve vegetation properties by a model inversion approach. Part A serves as introduction into the discrete scattering model and the role of the different vegetation parameters. Part B presents a sensitivity analysis of the simulated backscatter to the two main vegetation parameters this study is aiming to infer: shape and orientation of the vegetation inclusions. Part $\mathrm{C}$ details the retrieval methodology proposed to estimate both vegetation parameters from radar observations. Part D deals with direct application of the approach to data from the SMAP mission.

\section{A. Polarimetric Discrete Scatterer Model and the Role of the Vegetation Structure Parameters $A_{P}$ and $\psi$}

A coherent discrete scatterer model for a single-layer vegetation volume is presented in (1) using covariance matrix notation $\left[C^{V}\right][-]$. It is derived and explained in detail in [37, chapter 4.2.1.3], and is based on a vegetation model from Cloude [30]. This simplified discrete scatterer model, expressed with $\left[C^{V}\right]$ in single-channel form, simulates polarimetric vegetation scattering of a volume filled with evenly distributed and uniformly shaped spheroids within air as background medium. The model follows the single scattering approximation and does not include multiple scattering effects. Among the wide suite of available vegetation models, it is selected due to its low parameterization (two variables), but sufficient flexibility to represent diverse vegetation scattering at longer wavelengths (L- \& P-band) neglecting multiple scattering terms occurring at shorter frequencies (C- \& X-band).

$$
\left[C^{V}\right]=A \cdot\left[\begin{array}{ccc}
C_{11}^{V} & 0 & C_{13}^{V} \\
0 & C_{22}^{V} & 0 \\
C_{31}^{V} & 0 & C_{33}^{V}
\end{array}\right]
$$

with

$$
\begin{aligned}
& C_{11}^{V}=\frac{1}{8}\left(3 A_{P}^{2}+2 A_{P}+3+4\left(A_{P}^{2}-1\right) \operatorname{Sinc}(2 \psi)+\right. \\
& \left.\left(A_{P}-1\right)^{2} \operatorname{Sinc}(4 \psi)\right), \\
& C_{13}^{V}=\frac{1}{8}\left(A_{P}^{2}+6 A_{P}+1-\left(A_{P}-1\right)^{2} \operatorname{Sinc}(4 \psi)\right), \\
& C_{22}^{V}=\frac{1}{4}\left(A_{P}-1\right)^{2}(1-\operatorname{Sinc}(4 \psi)), \\
& C_{31}^{V}=\frac{1}{8}\left(A_{P}^{2}+6 A_{P}+1-\left(A_{P}-1\right)^{2} \operatorname{Sinc}(4 \psi)\right)^{*}, \\
& C_{33}^{V}=\frac{1}{8}\left(3 A_{P}^{2}+2 A_{P}+3-4\left(A_{P}^{2}-1\right) \operatorname{Sinc}(2 \psi)+\right. \\
& \left.\left(A_{P}-1\right)^{2} \operatorname{Sinc}(4 \psi)\right), \\
& A=\frac{1}{1+A_{P}^{2}},
\end{aligned}
$$

where $\operatorname{Sinc}(x)=\operatorname{Sin}(x) / x$ and '*' symbolizes the conjugate operator.

It considers different vegetation conditions expressed by the particle anisotropy $A_{p}[-]$ for the predominant shape of the main plant elements and by the orientation distribution width $\psi$ [rad] for different degrees of vegetation orientation from totally aligned to randomly oriented [38]. Fig. 1 provides a conceptual understanding of these two parameters for canonical vegetation structure cases [38]. A particle anisotropy of zero indicates vertically oriented dipoles, 
changing from vertical discs to spheres when increasing from zero to one. From one to infinity the shape changes from spheres to horizontal discs, ultimately reaching the shape of horizontal dipoles. An orientation distribution width of zero indicates completely aligned and oriented vegetation, while an increase of $\psi$ leads to randomization of the vegetation structure until a complete loss of orientation (complete randomization) for a distribution width of $90^{\circ}$.
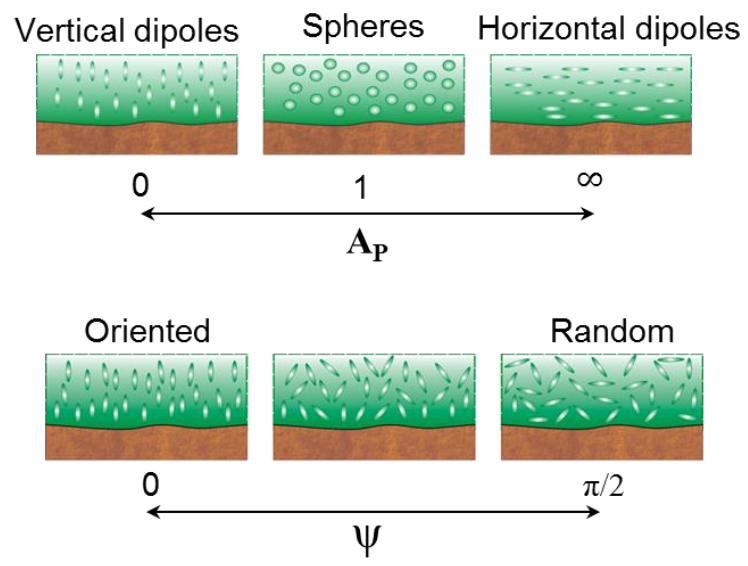

Fig. 1. Conceptual view on the vegetation structure parameters: particle shape $A_{p}$ (top) and orientation distribution width $\psi$ (bottom) [38].

The distribution of the different orientation angles is assumed to be uniform within $\psi$. Hence, the probability of occurrence for the individual orientation angles within $\psi$ is equal.

In order to obtain a relative measure, which is easier to model and invert compared to absolute terms, the vegetation volume model in (1) can be normalized with the cross-polarized backscattering component $\left|S_{P Q}\right|^{2}=\frac{1}{8}\left(A_{P}-1\right)^{2}(1-\operatorname{Sinc}(4 \psi))$ (half of $C_{22}^{V}$ in (1)) leading to (2).

Hence, $C_{\text {Norm }}^{V 11}$ and $C_{\text {Norm }}^{V 33}$ are the ratios of co- to crosspolarization intensity and a function of the model-based vegetation structure parameters $\left(A_{P}, \psi\right)$ :

$$
\begin{gathered}
C_{\text {Norm }}^{V 11}=\frac{\left|S_{H H}\right|^{2}}{\left|S_{H V}\right|^{2}}=\mu_{H H-H V}^{\text {Model }}= \\
\frac{\left(3 A_{P}^{2}+2 A_{P}+3+4\left(A_{P}^{2}-1\right) \operatorname{Sinc}(2 \psi)+\left(A_{P}-1\right)^{2} \operatorname{Sinc}(4 \psi)\right)}{\left(A_{P}-1\right)^{2}(1-\operatorname{Sinc}(4 \psi))} \\
C_{N \text { Norm }}^{V 33}=\frac{\left|S_{V V}\right|^{2}}{\left|S_{H V}\right|^{2}}=\mu_{V V-H V}^{M o d e l}= \\
\frac{\left(3 A_{P}^{2}+2 A_{P}+3-4\left(A_{P}^{2}-1\right) \operatorname{Sinc}(2 \psi)+\left(A_{P}-1\right)^{2} \operatorname{Sinc}(4 \psi)\right)}{\left(A_{P}-1\right)^{2}(1-\operatorname{Sinc}(4 \psi))}
\end{gathered}
$$

Eq. (3) and (4) can be applied as forward model formulas for co-to-cross-polarized backscatter ratios of vegetation, or socalled $\mu_{P P-P Q}^{M o d e l}$-parameters of the vegetation volume scattering model.

\section{B. Design of Sensitivity Study for $\mu_{P P-P Q}^{\text {Model }}$-Parameters with Respect to $A_{P}$ and $\psi$}

To investigate the sensitivity of $\mu_{P P-P Q}^{M o d e l}$-parameters to the vegetation parameters $\left(A_{p}, \psi\right)$, we design a forward modelling study. This leads to a deeper understanding of dependencies and to identify the best possible conditions for an observationbased parameter inversion. We will show the behavior and trends of $\mu_{P P-P Q}^{M o d e l}$ with fixing either $A_{p}$ or $\psi$ and varying the respective other parameter. This is done for a splitted $A_{p^{-}}$ range of zero to one and from one to $10^{8}$ due to different main orientation (either vertical or horizontal). The results of the sensitivity study are shown in section IV.A.

\section{Development of a Retrieval Methodology for Vegetation Parameters $\left(A_{P}, \psi\right)$}

Simplified vegetation models, like the one in (1), are implemented as standard components in model-based polarimetric decompositions for longer wavelengths to account for the vegetation scattering contribution [17], [30], [39]. Equation (5) shows the covariance matrices for a standard model-based decomposition architecture for vegetated agricultural soils [17]. It includes a soil surface scattering term $\left[C^{S}\right][-]$, a vegetation volume scattering term $\left[C^{V}\right][-]$ and an interacting soil-vegetation double bounce scattering term $\left[C^{D}\right][-][17],[39]$ :

$$
[C]=\left[C^{S}\right]+\left[C^{D}\right]+\left[C^{V}\right]
$$

This means in particular for a single co-polarized $[C]$-matrix element $\left|S_{P P}\right|^{2}[-]$ the decomposition can be specified as [39]:

$$
\left|S_{P P}\right|^{2}=f^{S}\left|S_{P P}^{S}\right|^{2}+f^{D}\left|S_{P P}^{D}\right|^{2}+f^{V}\left|S_{P P}^{V}\right|^{2},
$$

where the scattering matrix elements for the three polarimetric terms $\left|S_{P P}^{S}\right|^{2}, \quad\left|S_{P P}^{D}\right|^{2}$ and $\left|S_{P P}^{V}\right|^{2}$ are included with their respective lossy intensity components $f^{S}[-], f^{D}[-]$ and $f^{V}[-$ ].

In polarimetric decomposition theory for longer wavelengths such as L- and P-bands, it is assumed that vegetation volume scattering dominates the cross-polarized backscatter and the soil roughness contribution stays minor [17], [18], [30]. As $\left|S_{P Q}\right|^{2}[-]$ is indicative of volume scattering, co-polarized backscatter $\left|S_{P P}\right|^{2} \quad[-]$ can be corrected for a vegetation contribution $f_{V}\left|S_{P P}^{V}\right|^{2}$ by using the cross-polarized component together with a co-to-cross polarized projection using the backscatter ratio $\mu_{P P-P Q}[-]$ [30], [37]:

$$
\text { Using } \mu_{P P-P Q}\left|S_{P Q}\right|^{2}=f_{V}\left|S_{P P}^{V}\right|^{2} \text { for }
$$

$$
\left[C_{\text {Norm }}^{V}\right]=\frac{1}{8}\left(A_{P}-1\right)^{2}(1-\operatorname{Sinc}(4 \psi))\left[\begin{array}{ccc}
\frac{\left(3 A_{P}^{2}+2 A_{P}+3+4\left(A_{P}^{2}-1\right) \operatorname{Sinc}(2 \psi)+\left(A_{P}-1\right)^{2} \operatorname{Sinc}(4 \psi)\right)}{\left(A_{P}-1\right)^{2}(1-\operatorname{Sinc}(4 \psi))} & 0 & \frac{\left(A_{P}^{2}+6 A_{P}+1-\left(A_{P}-1\right)^{2} \operatorname{Sinc}(4 \psi)\right)}{\left(A_{P}-1\right)^{2}(1-\operatorname{Sinc}(4 \psi))} \\
0 & 2 & 0 \\
\frac{\left(A_{P}^{2}+6 A_{P}+1-\left(A_{P}-1\right)^{2} \operatorname{Sinc}(4 \psi)\right)^{*}}{\left(A_{P}-1\right)^{2}(1-\operatorname{Sinc}(4 \psi))} & 0 & \frac{\left(3 A_{P}^{2}+2 A_{P}+3-4\left(A_{P}^{2}-1\right) \operatorname{Sinc}(2 \psi)+\left(A_{P}-1\right)^{2} \operatorname{Sinc}(4 \psi)\right)}{\left(A_{P}-1\right)^{2}(1-\operatorname{Sinc}(4 \psi))}
\end{array}\right]
$$




$$
\begin{gathered}
f^{G}\left|S_{P P}^{G}\right|^{2}=f^{S}\left|S_{P P}^{S}\right|^{2}+f^{D}\left|S_{P P}^{D}\right|^{2}=\left|S_{P P}\right|^{2}-\mu_{P P-P Q}\left|S_{P Q}\right|^{2}= \\
\left|S_{P P}\right|^{2} \cdot\left(1-\mu_{P P-P Q} \cdot \frac{\left|S_{P Q}\right|^{2}}{\left|S_{P P}\right|^{2}}\right)
\end{gathered}
$$

where $f^{G}\left|S_{P P}^{G}\right|^{2} \quad[-]$ is the vegetation-corrected ground scattering term. As proposed in Jagdhuber et al. [40], the coto-cross-polarized backscatter ratio $\mu_{P P-P Q}$ will be linked to $\chi_{\mathrm{PP}-\mathrm{PQ}}[\mathrm{dB} / \mathrm{dB}]$ from (9) to (11). $\chi_{\mathrm{PP}-\mathrm{PQ}}$ is a ground backscatter-corrected (vegetation-only) co-to-cross-polarized backscatter ratio using the following assumption:

$$
\chi_{\mathrm{PP}-\mathrm{PQ}}=\frac{\sigma_{P P}^{G}-\sigma_{P P}}{\sigma_{P Q}}=\frac{\sigma_{P P}^{V}}{\sigma_{P Q}},
$$

where the backscatter of the vegetation (no ground contribution) $\sigma_{P P}^{V}[\mathrm{~dB}]$ is defined as $\sigma_{P P}^{V}=10 \cdot \log _{10}(1-$ $\left.\mu_{P P-P Q} \cdot \frac{\left|S_{P Q}\right|^{2}}{\left|S_{P P}\right|^{2}}\right)$ and $\sigma_{P P}^{G}$ is (8) transformed in decibel notation. In addition, $\sigma_{P Q}[\mathrm{~dB}]$ is the cross-polarized backscatter coefficients. After re-arranging (9) and algebraic modification, $\chi_{\mathrm{PP}-\mathrm{PQ}}$ and $\mu_{P P-P Q}$ are directly linked by:

$$
\chi_{\mathrm{PP}-\mathrm{PQ}} \cdot \log _{10}\left(\left|S_{P Q}\right|^{2}\right)=\log _{10}\left(1-\mu_{P P-P Q} \cdot \frac{\left|S_{P Q}\right|^{2}}{\left|S_{P P}\right|^{2}}\right)
$$

Solving for $\mu_{P P-P Q}$ leads to (11), which provides a direct retrieval of a vegetation-only co-to-cross-polarized backscatter ratio in linear units, if $\chi_{\mathrm{PP}-\mathrm{PQ}}$ is known [40].

$$
\mu_{P P-P Q}=\frac{\left|S_{P P}\right|^{2}}{\left|S_{P Q}\right|^{2}} \cdot\left(1-\left(\left|S_{P Q}\right|^{2}\right)^{\chi_{P P-P Q}}\right) .
$$

In an application case, (9) - (11) can be used to retrieve $\mu_{P P-P Q}$ from remote sensing data. Subsequently, the theoretical connection between $\mu_{P P-P Q}$ and the vegetation structure parameters $A_{p}$ and $\psi$ can be used for direct inversion of these parameters. During the inversion process, predicted $\mu_{P P-P Q}^{M o d e l}$ and observed $\mu_{P P-P Q}^{D a t a}$ have to be compared and a minimization procedure must be established. However, $\mu_{H H-H V}^{M o d e l}$ and $\mu_{V V-H V}^{\text {Model }}$ are unfortunately ambiguous with respect to particle anisotropy $A_{P} \quad\left(A_{p}=[0,1]\right.$ for vertical shapes \& $A_{p}=[1, \infty]$ for horizontal shapes), having a symmetry of both modelled $\mu_{P P-P Q}^{\text {Model }}$-values around $A_{P}=1$ (isotropic spherical shapes). This is shown in more detail by the results of the sensitivity analysis in section IV.A.

With this ambiguity of $\mu_{P P-P Q}^{M o d e l}$ regarding symmetry of $A_{P}$, the most suitable parameter estimation procedure is a two-step approach. First, the vegetation orientation $(\psi)$ retrieval is done for a fixed particle anisotropy of vertical $\left(A_{P}=0\right)$ and horizontal $\left(A_{P}=10000\right)$ dipoles for both polarization combinations (HH-HV \& VV-HV):

$$
\begin{gathered}
\phi_{\psi_{A_{P}=10000, \mu_{H H-H V}}}=\min \left|\mu_{H H-H V}^{D a t a}-\mu_{H H-H V}^{\text {Model }}\left(A_{P}=10^{4}, \psi\right)\right|, \\
\phi_{\psi_{A_{P}=10000, \mu_{V V-H V}}}=\min \left|\mu_{V V-H V}^{D a t a}-\mu_{V V-H V}^{\text {Model }}\left(A_{P}=10^{4}, \psi\right)\right|,
\end{gathered}
$$

$$
\begin{aligned}
\phi_{\psi_{A_{P}=0, \mu_{H H-H V}}} & =\min \left|\mu_{H H-H V}^{D a t a}-\mu_{H H-H V}^{\text {Model }}\left(A_{P}=0, \psi\right)\right|, \\
\phi_{\psi_{A_{P}=0, \mu_{V V-H V}}} & =\min \left|\mu_{V V-H V}^{D a t a}-\mu_{V V-H V}^{\text {Model }}\left(A_{P}=0, \psi\right)\right| .
\end{aligned}
$$

This ensures to include both major vegetation orientations (vertical and horizontal) in the retrieval.

In (12) the two solutions of $\psi\left(\psi_{A_{P}, \mu_{H H}-H V}, \psi_{A_{P}, \mu_{V V-H V}}\right)$ for the respective particle anisotropy (vegetation shape) $\left(A_{P}=\right.$ $10000, A_{P}=0$ ) have a split validity range around the $\mu_{P P-P Q}$-value of 3.0 due to the physics-given $A_{P}$-ambiguity of the retrieval (see Fig. 2a and [30]). Therefore, the following validity ranges are applied to generate the final two $\psi$ retrieval results $\left(\psi_{A_{P}=0}, \psi_{A_{P}=10000}\right)$ for vertical and horizontal dipoles:

In case of vertical dipoles $\left(A_{P}=0\right)$ this leads to the combined $\psi_{A_{P}=0}$-product:

$$
\begin{aligned}
\mu_{H H-H V}^{D a t a}<3.0 & \rightarrow \psi_{A_{P}=0, \mu_{H H-H V}} \\
\mu_{V V-H V}^{D a t a} \geq 3.0 & \rightarrow \psi_{A_{P}=0, \mu_{V V}-H V}
\end{aligned}
$$

In case of horizontal dipoles $\left(A_{P}=10000\right)$ this leads to the combined $\psi_{A_{P}=10000}$-product:

$$
\begin{aligned}
\mu_{H H-H V}^{D a t a} \geq 3.0 & \rightarrow \psi_{A_{P}=10000, \mu_{H H-H V}} \\
\mu_{V V-H V}^{D a t a}<3.0 & \rightarrow \psi_{A_{P}=10000, \mu_{V V-H V}}
\end{aligned}
$$

Consistently from both analysis, two results are produced: $\psi_{A_{P}=10000}$ and $\psi_{A_{P}=0}$.

The two data-derived co-to-cross polarized backscatter ratios $\left(\mu_{H H-H V}^{D a t a}, \mu_{V V-H V}^{D a t a}\right)$ are used in a second retrieval step to estimate the two $A_{P}$-products $\left(A_{P}^{H H-H V}, A_{P}^{V V-H V}\right)$, applying the assumption of a fixed $\psi$ of $90^{\circ}$ (random volume) to ensure the same model sensitivities to both polarization combinations (see Section IV.A), and at the same time confining the retrievable information only to the shape of the particles. No assessment on orientation of their major axes (i.e. vertical or horizontal dipoles) is possible anymore when $\psi$ is fixed to $90^{\circ}$ (cf. Fig. 2b):

$$
\begin{gathered}
\min _{A_{P}^{H H-H V}}\left|\mu_{H H-H V}^{D a t a}-\mu_{H H-H V}^{M o d e l}\left(A_{P}, \psi=90^{\circ}\right)\right|, \\
\min _{A_{P}^{V V}-H V}\left|\mu_{V V-H V}^{D a t a}-\mu_{V V-H V}^{M o d e l}\left(A_{P}, \psi=90^{\circ}\right)\right|,
\end{gathered}
$$

Two sets of $\psi$ - $\left(\psi_{A_{P}=10000}, \quad \psi_{A_{P}=0}\right)$ and $A_{P}$-values $\left(A_{P}^{H H-H V}, A_{P}^{V V-H V}\right)$ are obtained with the proposed retrieval. The two are thoroughly analyzed and compared with independent sources of vegetation height and biomass in Section IV.C.

\section{Adaption of Methodology for Application to Space-borne SMAP Data}

The methodology for vegetation parameter estimation developed in Section III is applied to global level-3 SMAP SAR data at L-band with fixed $40^{\circ}$ incidence angle. It is important to note that only radar intensity $\left(\left|S_{H H}\right|^{2},\left|S_{H V}\right|^{2}\right.$, $\left|S_{V V}\right|^{2}$ ) data and not fully polarimetric complex-valued measurements are provided from the active SMAP instrument. This is a challenging scenario, since phase information of the recorded microwaves would facilitate the retrieval. 
The pre-processed and filtered SMAP dataset is used to determine the data-based co-to-cross-polarized backscatter ratios $\left(\mu_{H H-H V}^{D a t a}, \mu_{V V-H V}^{D a t a}\right)$. It is important to mention that in the special case of SMAP $\chi_{\mathrm{PP}-\mathrm{PQ}}$ in Eq. (11) is assumed equivalent to $\Gamma_{P P-P Q}$ in the SMAP baseline algorithm developed by [45], [53]. Here, $\Gamma_{P P-P Q}$ is calculated as the 9 $\mathrm{km}$ (medium-scale) sub-pixel heterogeneity between co- and cross-polarization $\partial \sigma_{P P}$ and $\partial \sigma_{P Q}$ within one coarse-scale resolution cell $(36 \mathrm{~km})$ [46]. This leads to:

$$
\begin{array}{r}
\mu_{P P-P Q}=\frac{\left|S_{P P}\right|^{2}}{\left|S_{P Q}\right|^{2}} \cdot\left(1-\left(\left|S_{P Q}\right|^{2}\right)^{\chi_{P P-P Q}}\right) \cong \\
\frac{\left|S_{P P}\right|^{2}}{\left|S_{P Q}\right|^{2}} \cdot\left(1-\left(\left|S_{P Q}\right|^{2}\right)^{\Gamma_{P P-P Q}}\right) .
\end{array}
$$

Hence, the sub-pixel spatial heterogeneity, expressed by the covariation of co- and cross-polarized backscatter $\Gamma_{P P-P Q}$ in the coarse-scale cell, is taken to be indicative for the amount of vegetation-only co-to-cross-polarized backscatter ratio $\mu_{P P-P Q}$. Behind is the assumption that local spatial heterogeneity, expressed by $\Gamma_{P P-P Q}$, for one time instant is dominated by vegetation [40], [45], [49], [53]-[55].

\section{RESUlts OF VEGETATION PARAMETER RETRIEVAL USING SMAP DATA}

The results of the sensitivity analysis, the application to SMAP data and the comparison of the retrieved vegetation structure parameters with independent data sets of vegetation height and above ground biomass are presented in the following.

\section{A. Sensitivity Analysis of $\mu_{P P-P Q}^{M o d e l}$-Parameters with Respect to} Vegetation Structure Parameters $A_{P}$ and $\psi$

It is important to understand from Fig. 2a that the dependencies of $\mu_{H H-H V}^{M o d e l}$ and $\mu_{V V-H V}^{M o d e l}$ on the orientation angle distribution width $\psi$ are equivalent, when either vertical
$\left(A_{p}=0\right)$ or horizontal $\left(A_{p}=10000\right)$ dipoles are assumed as particle shapes.

Note if particle shapes closer to spheres $\left(A_{p} \rightarrow 1\right)$ are assumed, the congruent course of the two $\mu_{P P-P Q}^{\mathrm{Model} \text {-curves in }}$ Fig. 2a would not be given and the dependencies would differ significantly (not shown). This is why assuming dipoles as particle shape of the vegetation volume is crucial: the identical $\mu_{P P-P Q}^{M o d e l}$-trend for both dipole types enables a retrieval of the orientation distribution width $\psi$ using only one $\mu_{P P-P Q}-$ value. The value of the respective $\mu_{P P-P Q}^{M o d e l}$ informs on whether the main shape of the plant element has a horizontally $\left(\mu_{P P-P Q}^{M o d e l}=\right.$ $[0,3]$ ) or vertically (from $\mu_{P P-P Q}^{M o d e l}=[3, \infty[$ ) oriented major axis or (see Fig. 2a).

The dependence of $\mu_{P P-P Q}^{M o d e l}$ on the shape of the main plant element $A_{p}$ is depicted in Fig. $2 \mathrm{~b}$ for three vegetation volume orientations $\left(\psi=\left[10^{\circ}, 45^{\circ}, 90^{\circ}\right]\right)$. If a randomly oriented volume $\left(\psi=90^{\circ}\right)$ is assumed, then $\mu_{H H-H V}^{M o d e l}$ and $\mu_{V V-H V}^{M o d e l}$ have the same dependencies within an $A_{p}$-range from zero (vertically oriented dipoles) to one (spheres) or from one to infinity (horizontally oriented dipoles). The more the vegetation volume is oriented $\left(\psi=10^{\circ}\right)$, the more the curves

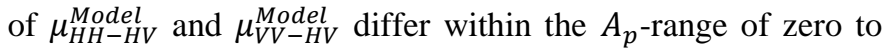
one due to different interaction of the polarized EM-waves with oriented (anisotropically scattering) vegetation. This is especially pronounced for low $A_{p}$-values. For $A_{p}$ around 1 (spheres), $\mu_{P P-P Q}^{M o d e l}$-values rise towards infinity.

Hence, for the $A_{p}$-range from one (spheres) towards positive infinity (horizontal dipoles) the $\mu_{P P-P Q}^{M o d e l}$-values decrease monotonically, as depicted in Fig. 2c. The sensitivity of $\mu_{P P-P Q}^{M o d e l}$ for $A_{p}$ is lost at $A_{p}$-values around 1000 and therefore an $A_{p}$-value of 10000 is already representative for horizontal dipole. However, this advocates $A_{p}$-retrievals to be conducted within the range of zero (vertical dipoles) to one (spheres) due to the higher sensitivity of $\mu_{P P-P Q}^{M o d e l}$.

b)

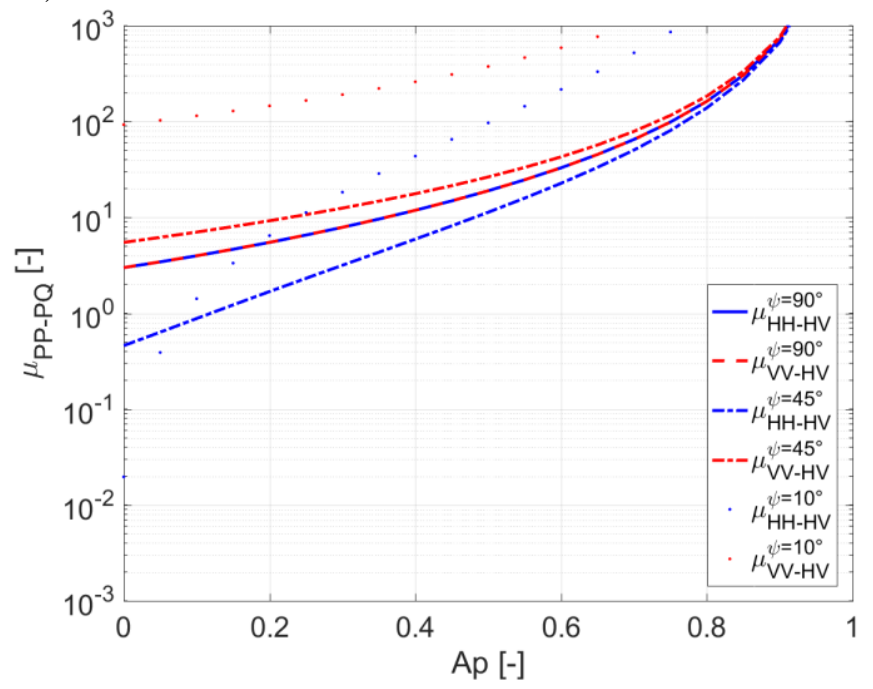




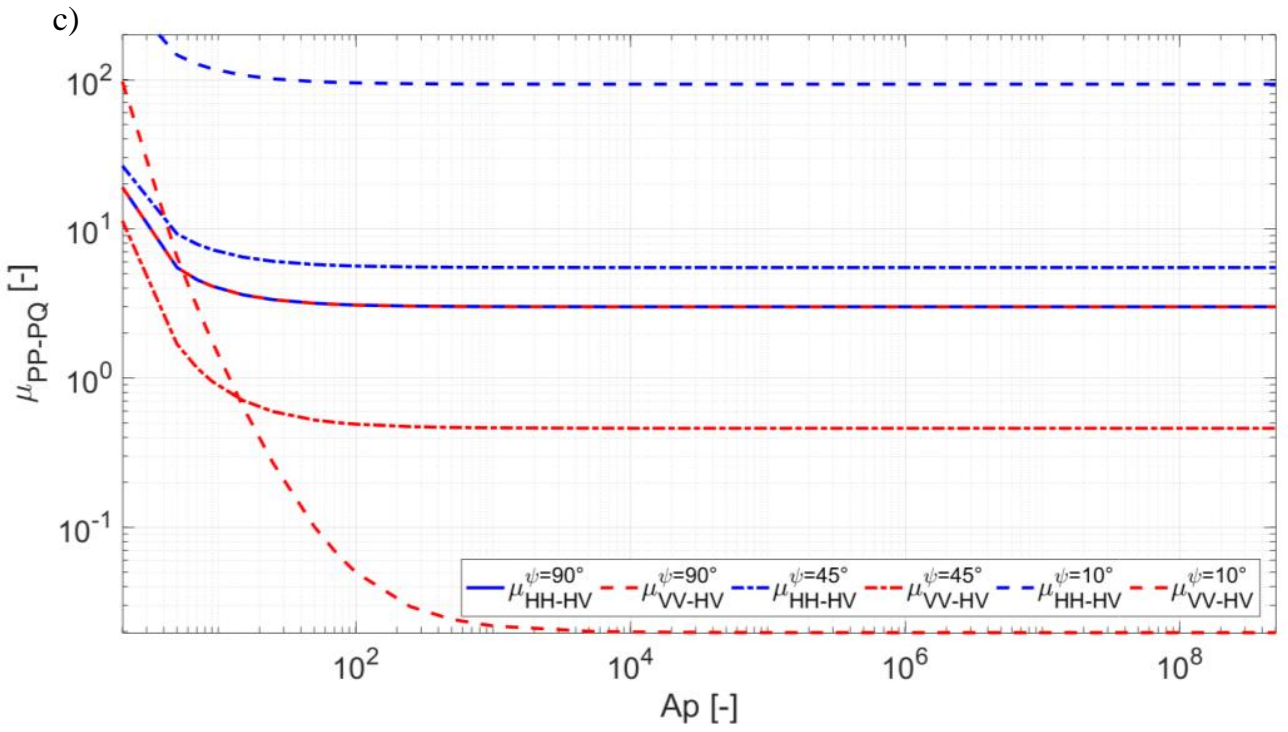

Fig. 2. Modelled vegetation-only co-to-cross-polarized backscatter ratio $\mu_{P P-P Q}^{M o d e l}[-]$ for different assumptions concerning vegetation shape $\left(A_{p}\right)$ or orientation distribution $(\psi)$; a) $\mu_{P P-P Q}^{M o d e l}[-]$ for vertical $\left(A_{p}=0\right)$ and horizontal $\left(A_{p}=10000\right)$ shapes along orientation distribution width $\psi$ from absolutely oriented $\left(\psi=0^{\circ}\right)$ to randomly oriented $\left(\psi=90^{\circ}\right)$; b) $\mu_{P P-P Q}^{\text {Model }}[-]$ for strongly oriented $\left(\psi=10^{\circ}\right)$, half-randomly $\left(\psi=45^{\circ}\right)$ until totally randomly oriented $\left(\psi=90^{\circ}\right)$ vegetation along vegetation shape $A_{p}$ from vertical dipoles $\left(A_{p}=0\right)$ until spheres $\left(A_{p}=1\right)$; c) $\mu_{P P-P Q}^{\text {Model }}[-]$ for strongly oriented $\left(\psi=10^{\circ}\right)$, half-randomly $\left(\psi=45^{\circ}\right)$ until totally randomly oriented $\left(\psi=90^{\circ}\right)$ vegetation along vegetation shape $A_{p}$ from spheres $\left(A_{p}=1\right)$ towards horizontal dipoles $\left(A_{p}=5.0 \cdot 10^{8}\right)$

\section{B. Estimation of vegetation structure parameters from global SMAP Radar Data}

In this section the developed method is applied on SMAP radar data for global analysis and for detailed regional analysis of vegetation structure parameters. The initial (non-vegetationfiltered) input radar backscatter intensities $[\mathrm{dB}]$ are shown in Fig. 3 averaged over the SMAP active-passive acquisition period (04-07/2015).

a)

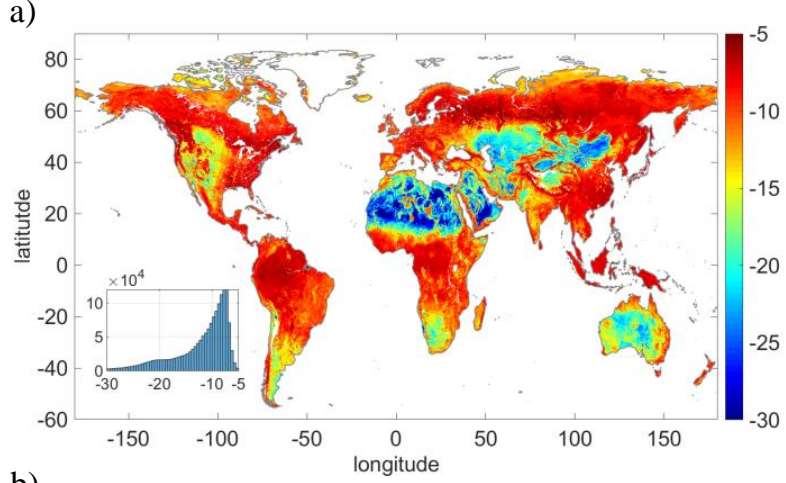

b)

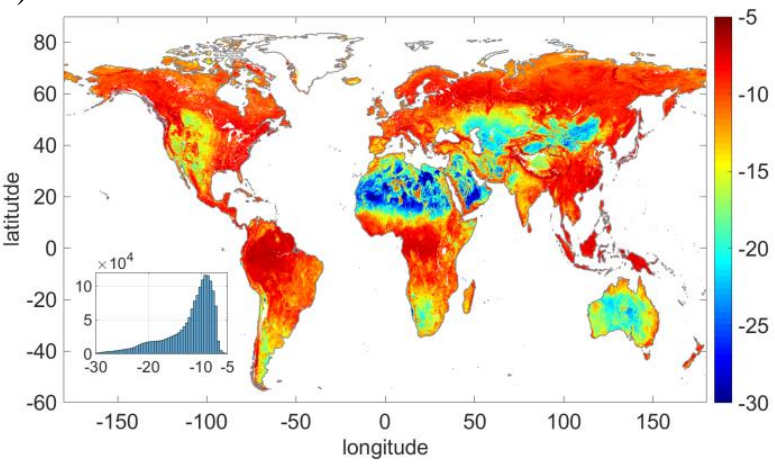

c)

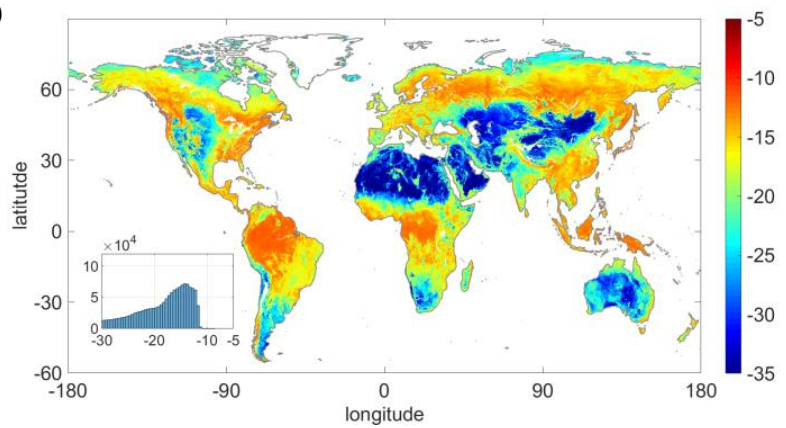

Fig. 3. Global comparison of measured, time-averaged SMAP backscatter intensities [dB]; a) $\left|S_{H H}\right|^{2}$; b) $\left|S_{V V}\right|^{2}$; c) $\left|S_{H V}\right|^{2}$; Averaging was done over the SMAP active-passive acquisition period (04-07/2015); inset shows histogram of respective backscatter intensity.

They serve as basis for the subsequent estimation of vegetation structure parameters. The backscatter variations follow mainly the global land cover patterns from the tropical to the boreal and Tundra zones with low backscatter especially in arid and hyper-arid (deserted) regions. The co-polarized backscatter intensities $(\mathrm{HH}, \mathrm{VV})$ indicate distribution maxima around $-10 \mathrm{~dB}$, while the cross-polarized intensity (HV) has typically a lower distribution maximum around $-15 \mathrm{~dB}$ (see inset histograms in Fig. 3).

\section{1) Global Analysis of Retrieved Vegetation Structure Parameters}

The developed vegetation parameter estimation method of Section III is applied to the space-borne SMAP data introduced in Section II. Both vegetation-only co-to-crosspolarized backscatter ratios $\mu_{H H-H V}^{D a t a}$ and $\mu_{V V-H V}^{D a t a}$ are displayed in Figs. $4 a$ and $4 b$, together with the illustration of their differences in Figs. 4c and 4d. Figure 4d reveals a difference 
for the two polarization combination with $\mu_{V V-H V}^{D a t a}$ having the lower maximum of both $\mu^{\text {Data }}$-parameters.

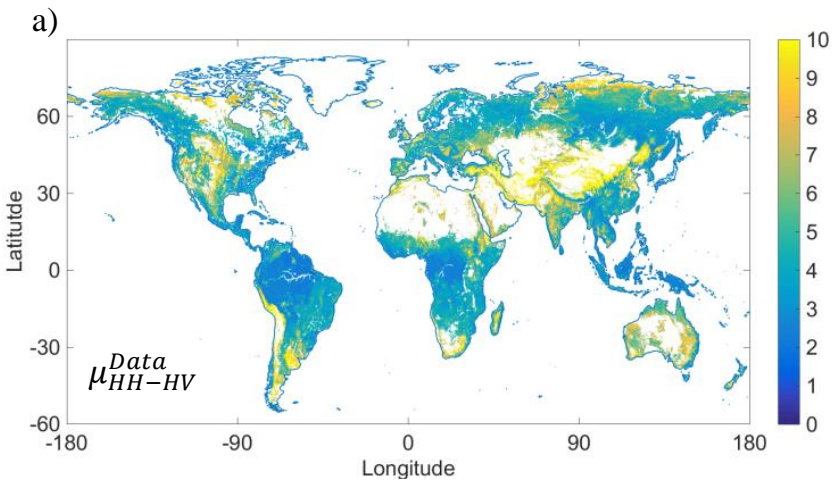

$$
\text { b) }
$$
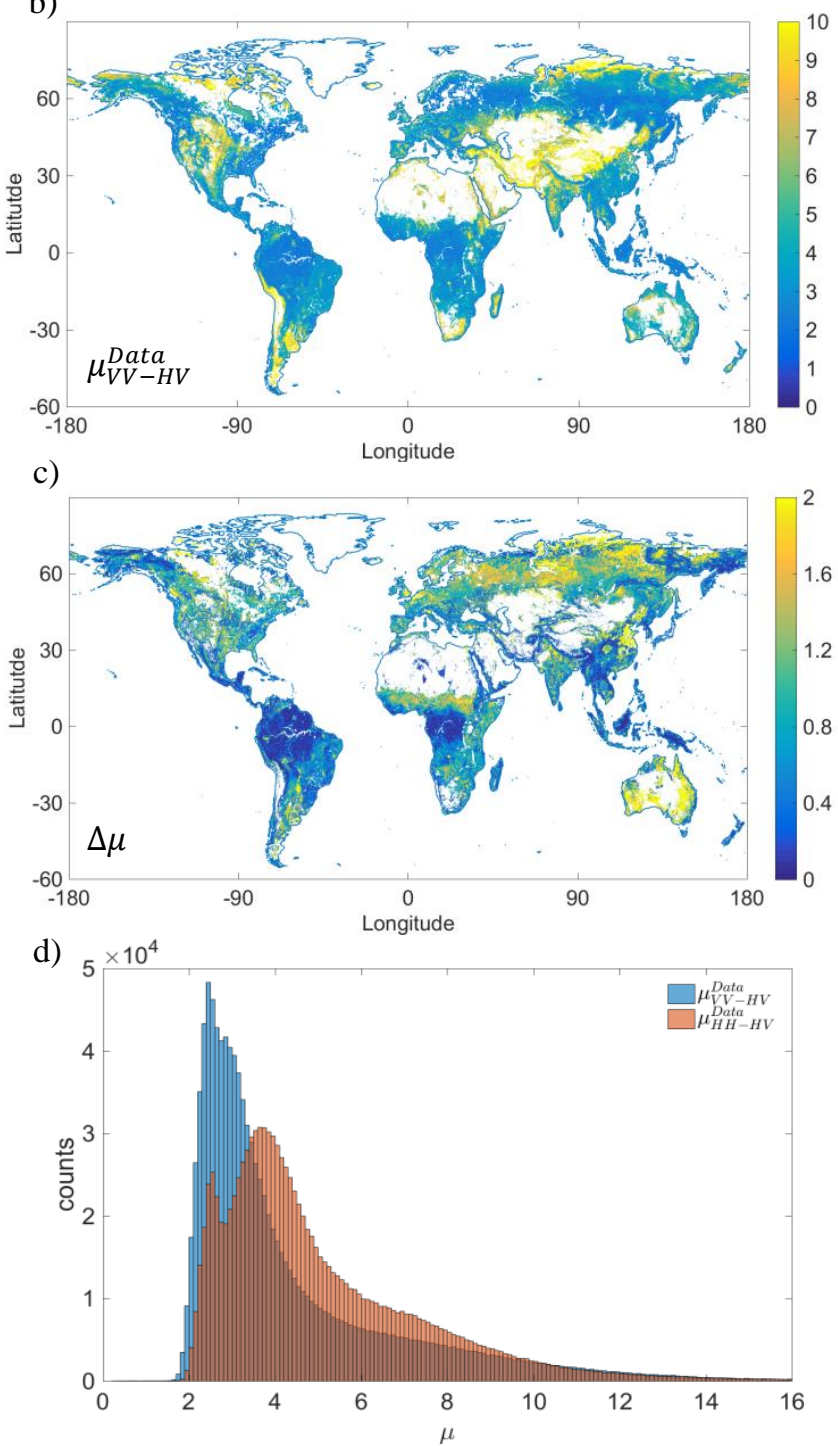

Fig. 4. Global comparison of retrieved, time-averaged vegetation-only co-tocross-polarized backscatter ratio $\mu_{P P-P Q}[-]$; a) $\mu_{H H-H V}^{D a t a}$; b) $\mu_{V V-H V}^{D a t a}$; c) Absolute difference of both: $|\Delta \mu|=\left|\mu_{V V-H V}^{D a t a}-\mu_{H H-H V}^{D a t a}\right|$; d) Histogram of $\mu_{V V-H V}$ and $\mu_{H H-H V}$; Averaging was done over the SMAP active-passive acquisition period (04-07/2015) and blank land masses are masked nonvegetated areas (see Fig. 4) or invalid retrievals.

The most significant absolute difference, $|\Delta \mu|$ shown in Fig. 4c, occurs in semi-arid areas, (e.g. in the Sahel zone, Australia and Tundra regions) where small-scale spatial heterogeneity is distinct. In contrast, the highest similarity of both $\mu^{\text {Data }}$ parameters is at the tropical and subtropical regions, where the structure is complex, but spatially more homogeneous. Thus, both polarimetric retrievals lead to similar results.

Using the methodology presented in Section III leads to the model-based vegetation structure parameters: orientation distribution width $(\psi)$ and particle anisotropy $\left(A_{P}\right)$. Since the algorithm is executed as a two-step process, the orientation distribution width $\psi$ with pre-defined $A_{P}$-values (vertical dipoles: $A_{P}=0$, horizontal dipoles: $\left.A_{P}=10000\right)$ are presented first and afterwards the particle anisotropy $A_{P}$ with a fixed $\psi-$ value of $90^{\circ}$. Fig. 5 shows the global comparison of the estimated vegetation orientation distribution width $\psi$ averaged for the SMAP active-passive acquisition period (04-07/2015), while non-vegetated areas are masked out.

Figs. 5a and 5b compare the global $\psi$-estimation for vertical and horizontal dipoles, both fixed inputs representing the shape of the main plant element. For randomly oriented volumes (tropical rainforest, temperate \& boreal forest zones) both $\psi$-products indicate high values above $80^{\circ}$. Lower $\psi$ values $\left(40^{\circ}<\psi<80^{\circ}\right)$ are found within the global Savanna and grassland zones. Lowest values $\left(\psi<40^{\circ}\right)$ are present close to arid (Sahara) and barren (Himalayas) regions.

In addition, Fig. 5c reveals the absolute difference $|\Delta \psi|$ between the two $\psi$-estimates. Small differences between $\psi_{A_{P}=0}$ and $\psi_{A_{P}=10000}$ are found in highly vegetated areas of the tropical vegetation belt where the complexity of the canopy cover lead to a random orientation in the model-based $\psi$-estimation. Thus, the shape of the constituting particles is not an influential factor any more. This is different for the boreal and agricultural zones. Here the reduced complexity in vegetation structure (open forest) and the stronger orientation of crops (stalk- or leave-dominated plant structures) lead to significant differences between $\psi_{A_{P}=0}$ and $\psi_{A_{P}=10000}$. However the majority of $|\Delta \psi|$-values stays below $15^{\circ}$ (1/6 of value range), which indicates a certain degree of similarity. The histograms for both $\psi$-products in Fig. 5 d expose similar patterns for the two retrievals and bi-modal distributions with local maxima between $35^{\circ}$ and $65^{\circ}$ as well as between $80^{\circ}$ and $90^{\circ}$. There exist no values lower than $10^{\circ}$ and only few below $20^{\circ}$. This means absolutely aligned vegetation structures, occurring most likely in agricultural areas, could not be detected, which might be due to the coarse grid of the SMAP radar sensor ( $9 \mathrm{~km}$, aggregated from $3 \mathrm{~km}$ ) with respect to the appearance and extent of strongly aligned vegetation within a kilometer-wide resolution cell.

Fig. 6 displays double (red: $\psi_{A_{P}=0}$, blue: $\psi_{A_{P}=10000}$ ) box plots for the different IGBP-based land cover classes (excluding urban, snow-covered and barren regions). Forests are located on a high level of $\psi$ between $60^{\circ}$ and $90^{\circ}$, while crop-, wet- $\&$ grassland as well as open shrubs remain on a lower level predominantly between $30^{\circ}$ and $60^{\circ}$. Closed shrubs, (woody) savanna and other natural vegetation exhibit intermediate levels of $\psi$ with $50^{\circ}$ and $80^{\circ}$. Looking more closely to the difference between $\psi_{A_{P}=0}$ and $\psi_{A_{P}=10000}$ reveals that especially the evergreen and deciduous needle-leaf forests as well as closed shrub land have distinctively different distribution width, when assuming different initial particle 
shapes. Hence, assumed vertical shapes $\left(A_{P}=0\right)$ lead to stronger estimated orientation, meaning smaller distribution

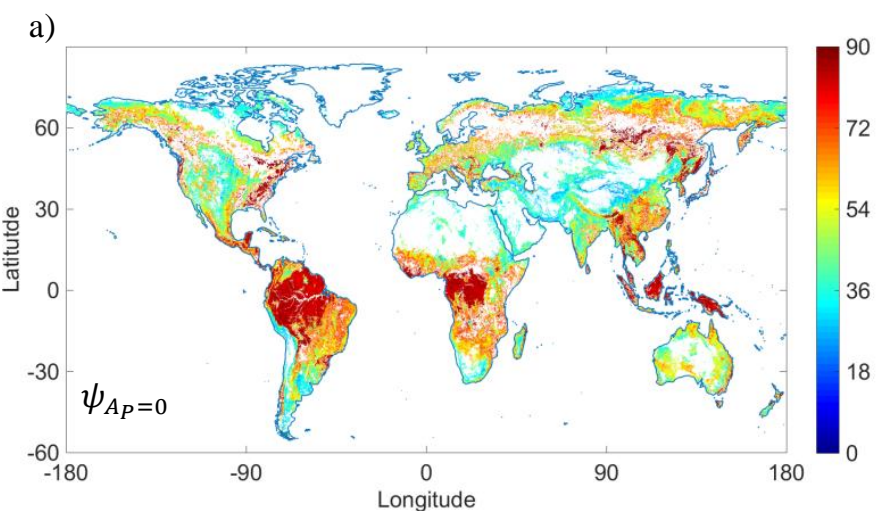

b)

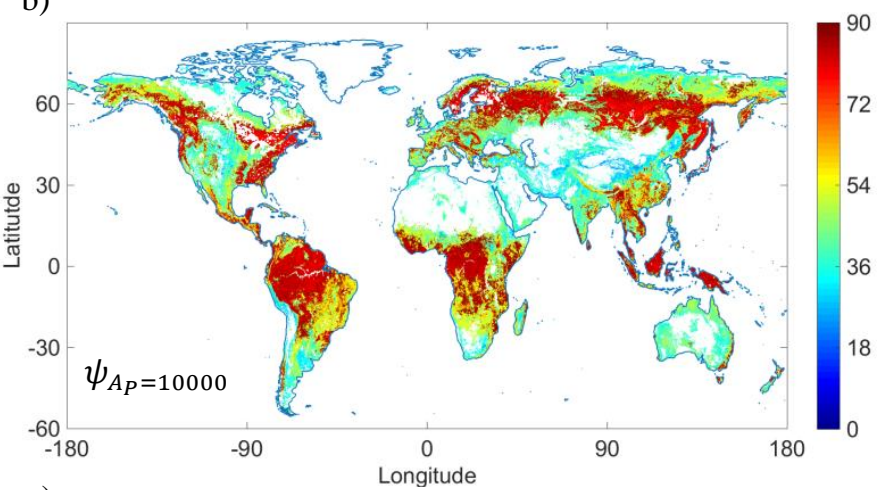

c)

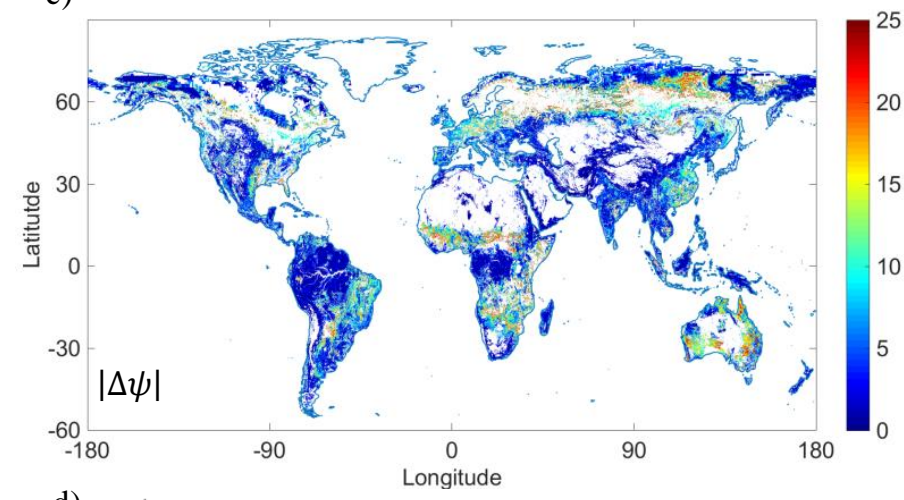

d) $\times 10^{4}$

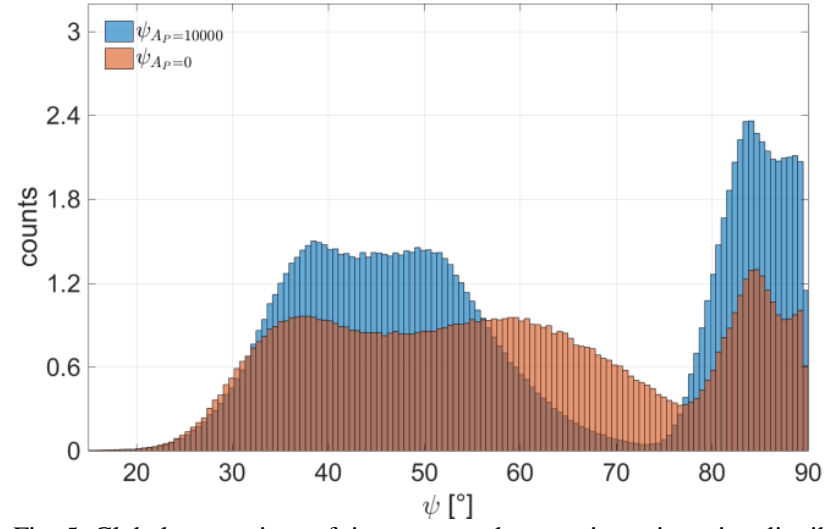

Fig. 5. Global comparison of time-averaged vegetation orientation distribution width $(\psi)\left[{ }^{\circ}\right]$ : a) Taking vertical dipoles $\left(A_{P}=0\right) \psi_{A_{P}=0} ;$ b) Taking horizontal dipoles $\left(A_{P}=10000\right) \quad \psi_{A_{P}=10000} ;$ c) Difference of both: $|\Delta \psi|=$ $\left|\psi_{A_{P}=0}-\psi_{A_{P}=10000}\right|$; Histogram of $\psi_{A_{P}=0}$ and $\psi_{A_{P}=10000}$; Averaging was done over the SMAP active-passive acquisition period (04-07/2015) and blank land masses are masked non-vegetated areas (see Fig. 4) or invalid retrievals. width $\psi$.

Moreover, the box plots for $\psi_{A_{P}=10000}$ (horizontal dipoles) unveil for woody savanna and closed shrub lands a wide range of $\psi$ from $50^{\circ}$ to $85^{\circ}$ for the interquartile period. This might be due to the variety in vegetation structure cases allocated to these land cover classes.

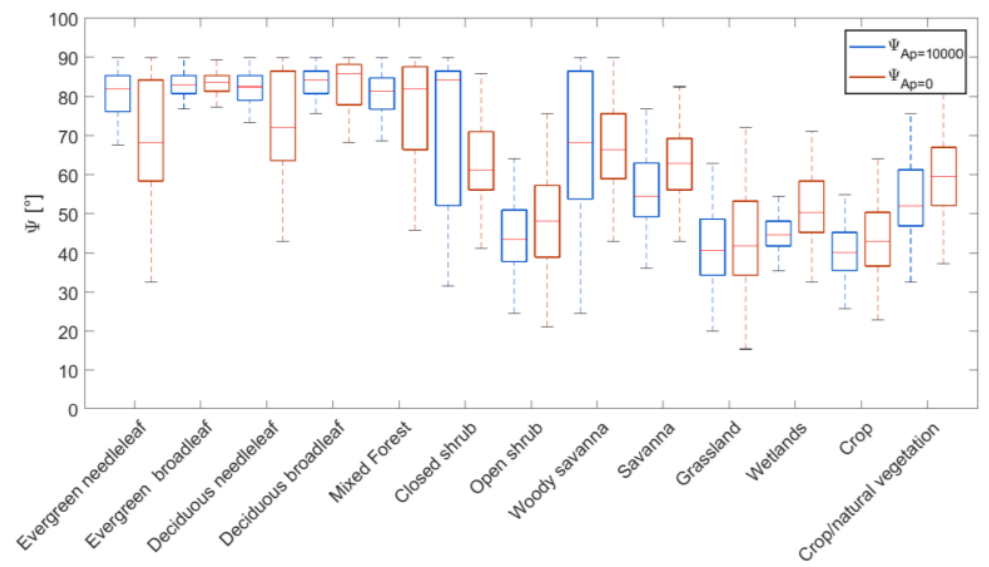

Fig. 6. Box plots of retrieved time-averaged vegetation orientation distribution width $(\psi)\left[^{\circ}\right]$ where red color indicates the assumption of vertical dipoles $\left(A_{P}=0\right)$ and blue color the assumption of horizontal dipoles $\left(A_{P}=10000\right)$; Box plots are displayed for different IGBP-based land cover classes (excluding urban, cold and barren regions); The red horizontal line within each box indicates the median value; The box represents the interquartile region $(25 \%-75 \%)$ and the whiskers show the extent to the minimum and maximum value of $\psi$.

The particle anisotropy $A_{P}$ was retrieved globally assuming a fixed $\psi$-value of $90^{\circ}$ and is shown in Fig. 7 for the two polarization combinations $(H H-H V, V V-H V)$. The range of possible values stretches from zero, which indicates dipoles, via disks to spheres for an $A_{P}$-value of one. Figs. 7a and $7 \mathrm{~b}$ reveal that most of the values are close to zero and none exceeds 0.6 (disks).

More in detail, dipole-representing values are dominant and can be found in the tropical, temperate and boreal forest regions. In contrast, higher values $\left(A_{P}>0.3\right)$ can be found close to mountainous and tundra regions, whereby (semi-) arid regions do not necessarily exhibit the highest values (cf. Australia). The distribution of values for $A_{P}^{H H-H V}$ and $A_{P}^{V V-H V}$ in Fig. $7 \mathrm{~d}$ support this observations and indicate the same range of values for both retrievals having just a different distribution from 0.01 until 0.35 . The difference of both retrievals $\Delta A_{P}$ is visualized in Fig. $7 \mathrm{c}$, where strongest deviations occur for Savanna, crop- and shrublands as well as for evergreen and deciduous needle leaf forests mainly in higher Northern latitudes.

Fig. 8 presents double (red: $A_{P}^{V V-H V}$, blue: $A_{P}^{H H-H V}$ ) box plots for different IGBP-based land cover classes (excluding urban, cold and barren regions). For forested areas, closed shrub regions and woody savannas with distinct vegetation cover (60\% and higher) $A_{P}$-values with a median below 0.1 (dipoletype of shape) are predominantly encountered. In addition, open shrub, crops, grass- and wetlands show median values around 0.2 (more disk-like shape). $A_{P}$-values above 0.3 occur only sparsely indicating the absence of sphere-like scattering. Moreover, the retrieval difference between $A_{P}^{V V-H V}$ and 
$A_{P}^{H H-H V}$ is mostly above 0.05 except for evergreen as well as deciduous broadleaf forest and grassland, where directionali-

a)

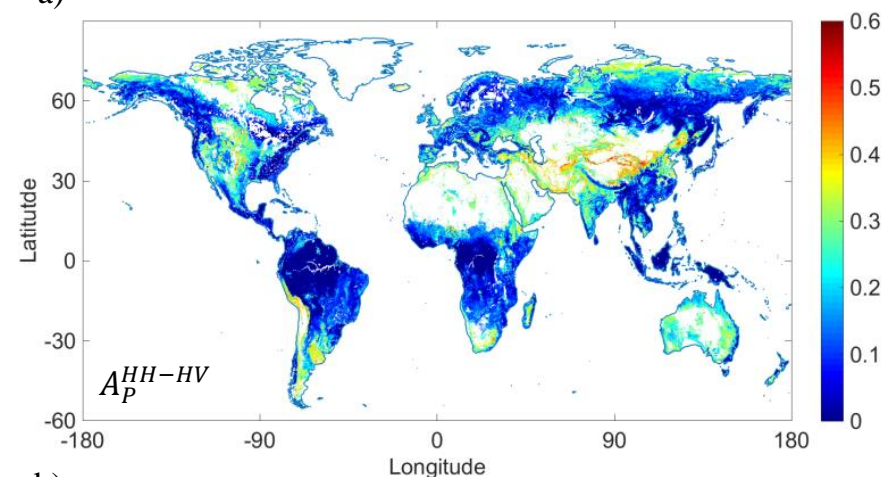

b)
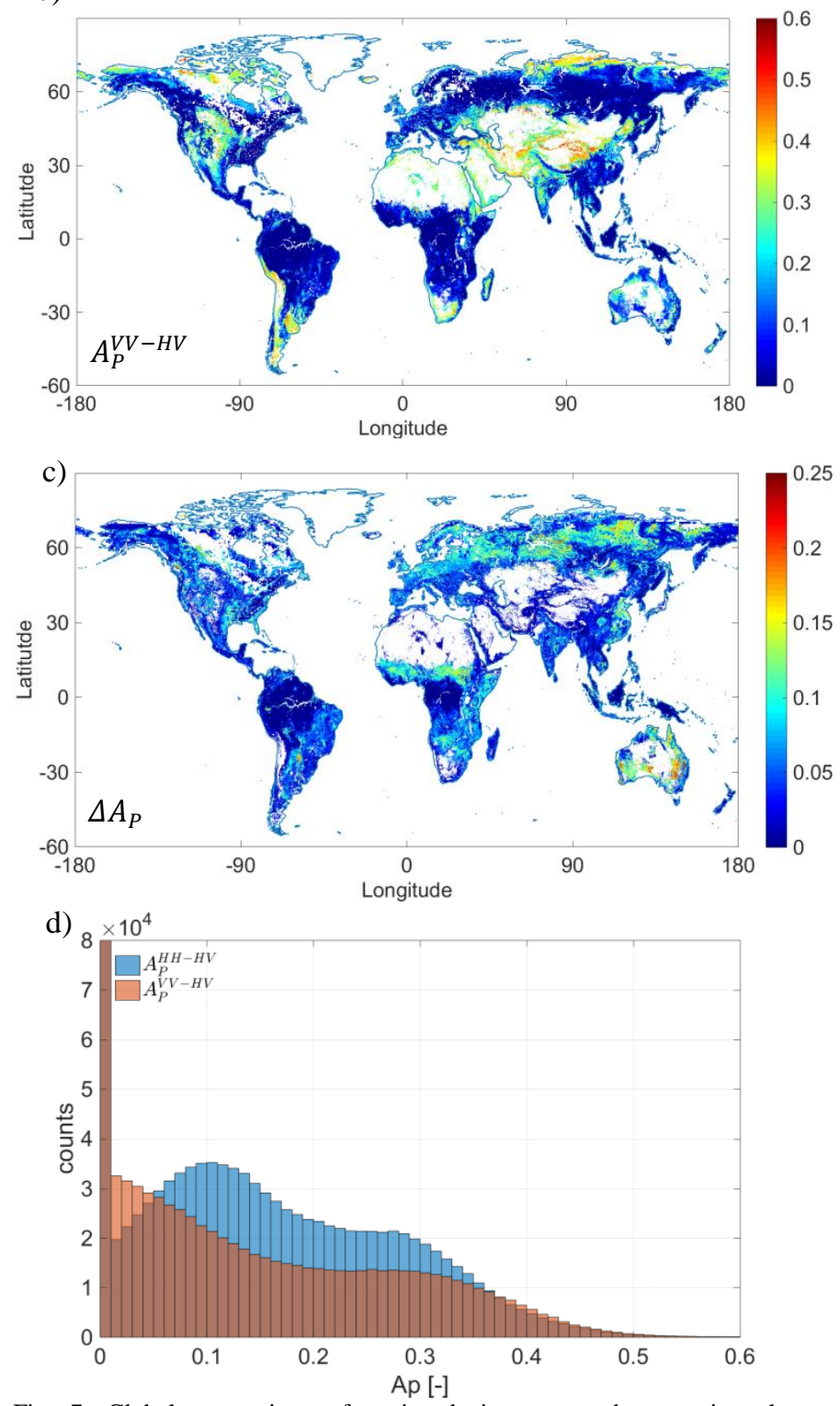

Fig. 7. Global comparison of retrieved time-averaged vegetation shape (particle anisotropy) $A_{P}[-]$ assuming a randomly oriented canopy $\left(\psi=90^{\circ}\right)$ : a) $A_{P}^{H H-H V}$ from $\mu_{H H-H V}$; b) $A_{P}^{V V-H V}$ from $\mu_{V V-H V}$; c) Difference of both: $\Delta A_{P}=$ $\left|A_{P}^{V V-H V}-A_{P}^{H H-H V}\right|$; d) Histogram of $A_{P}^{V V-H V}$ and $A_{P}^{H H-H V}$; Averaging was done over the SMAP active-passive acquisition period (04-07/2015) and blank land masses are masked non-vegetated areas (see Fig. 4) or invalid retrievals. ties within the vegetation structure do not lead to polarizationinduced differences within the $A_{P}$-retrieval.

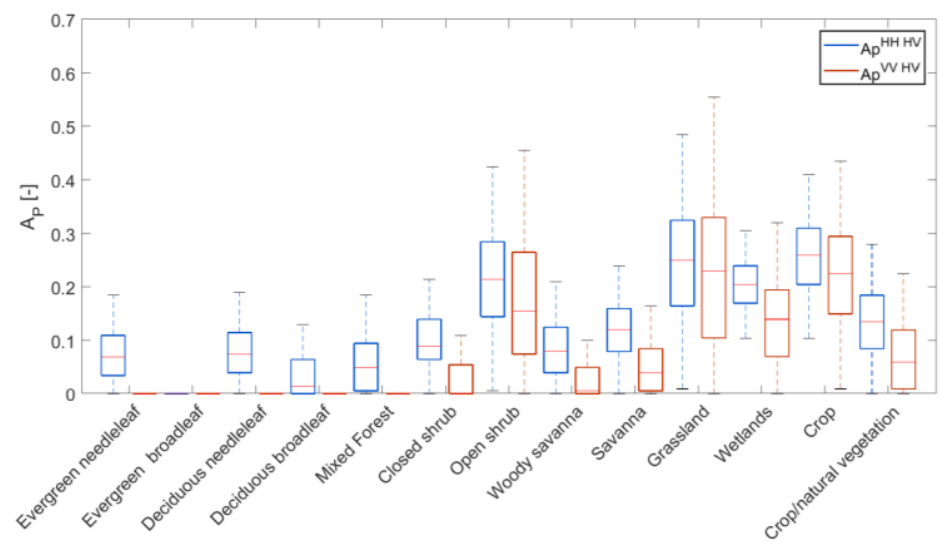

Fig. 8. Box plots of retrieved time-averaged vegetation shape (particle anisotropy) $\left(A_{P}\right)[-]$ where red color indicates the derivation from $\mu_{V V-H V}$ and blue color the derivation from $\mu_{H H-H V}$; Box plots are displayed for different IGBP-based land cover classes (excluding urban, cold and barren regions); The red horizontal line within each box indicates the median value; The box represents the interquartile region $(25 \%-75 \%)$ and the whiskers show the extent to the minimum and maximum value of $A_{P}$.

\section{2) Analysis of Retrieved Vegetation Structure Parameters for Different Regions of Interest}

In order to gain detailed insights on the dependencies between vegetation conditions and the retrieved model-based vegetation structure parameters $A_{P}$ and $\psi$, six regions of interest with spatially homogenous land cover were selected (see Fig. 9a). The exemplary regions feature different regimes for vegetation cover and plant moisture, as indicated by radar vegetation index $(R V I)$ in Fig. 9b [56], [57] and vegetation water content $(V W C)\left[\mathrm{kg} / \mathrm{m}^{2}\right]$ in Fig. 9c which was provided from SMAP-mission auxiliary data of [42]. The conditions stretch from high cover $(R V I \sim 1.0)$ and moisture $(V W C>9$ $\mathrm{kg} / \mathrm{m}^{2}$ ) within tropical rainforest of the Congo basin to no cover $(R V I \sim 0.2)$ and no moisture $\left(V W C<0.1 \mathrm{~kg} / \mathrm{m}^{2}\right)$ within the Sahara and the Arabian Desert. In the analysis, savanna and grassland regions serve as reference with equivalent environmental conditions $\left(R V I \sim 0.3, V W C \sim 0.2 \mathrm{~kg} / \mathrm{m}^{2}\right)$, where only little deviations between retrieved vegetation structure parameters are anticipated.

Fig. 10 displays box-plots of the retrieve results for the timeaveraged shape $A_{P}$ and vegetation orientation distribution width $\psi$ of the vegetation from the six regions of interest. The most significant difference for both parameters occurs between the regions of forest-size vegetation cover (tropical rainforest and woody savanna) and the agriculture-size vegetated areas (agriculture, savanna, grassland and barren). In Fig. 10a, $A_{P}$ stays below 0.2 for the forest-size vegetation and ranges mainly between 0.2 and 0.4 for agriculture-size plants. In addition, retrieved $\psi$-values in Fig. $10 \mathrm{~b}$ vary between $50^{\circ}$ and $90^{\circ}$ for taller (forest) vegetation due to complex canopy structures and between $20^{\circ}$ and $50^{\circ}$ for agricultural types revealing more oriented and less random vegetation structures. 
a)

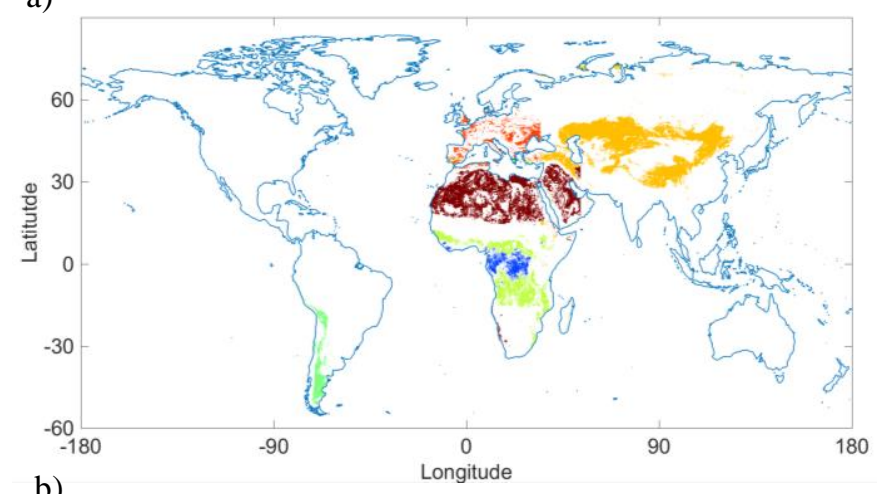

b)

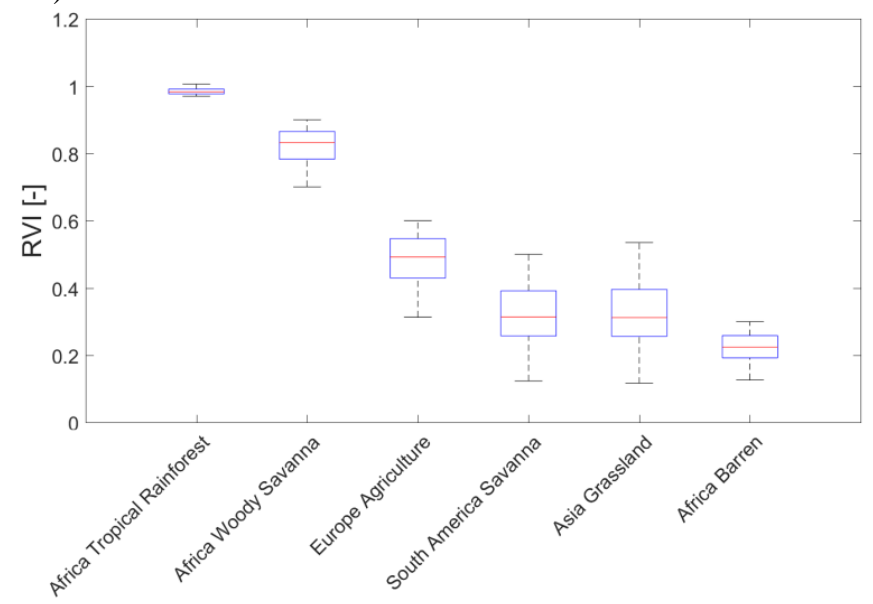

c)

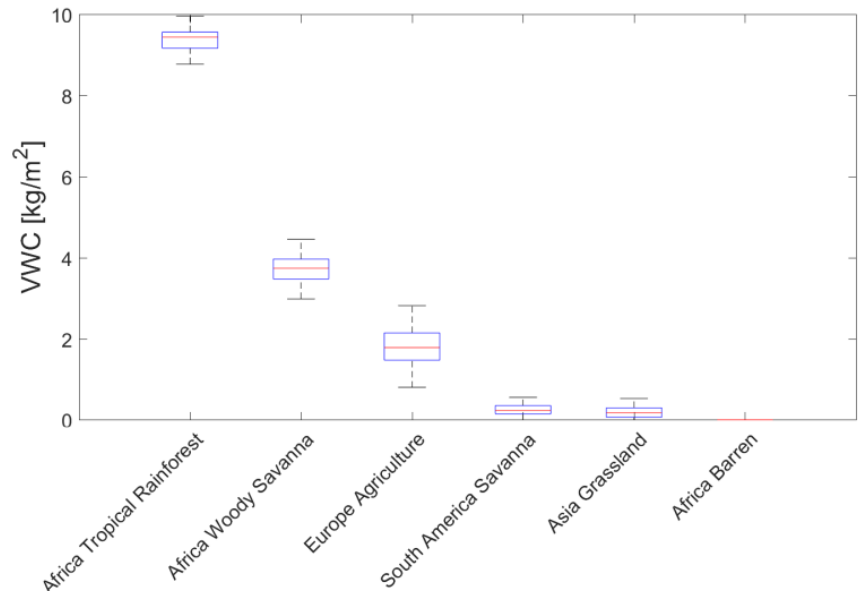

Fig. 9. a) Global overview of the IGBP-based regions of interest for analysis of the developed vegetation parameter retrieval; Blue: tropical rain forest (Africa), light green: woody savanna (Africa), red: agriculture (Europe), green: savanna (South America), orange: grassland (Asia), dark red: barren land (Africa); b) Vegetation cover indicated by the time-averaged radar vegetation index (RVI) [-] calculated according to [56], [57]; c) Plant moisture described by time-averaged vegetation water content $\left(V W C\left[\mathrm{~kg} / \mathrm{m}^{2}\right]\right)$ provided by [42]; The red horizontal line within each box indicates the median value; The box represents the interquartile region $(25 \%-75 \%)$ and the whiskers show the extent to the minimum and maximum value of $R V I[-]$ and $V W C\left[\mathrm{~kg} / \mathrm{m}^{2}\right]$, respectively.

The difference between the two results of $A_{P}$ and $\psi$, given for each respective class due to polarization for $A_{P}$ and shape assumption for $\psi$, is only minor (equivalent median-values in Fig. 10), except for the woody savanna region in Africa and the agricultural region in Europe. Moreover, the two control classes savanna and grassland state stable retrieval conditions by showing the same average estimation result (similar median for both retrievals), but grassland exhibits a wider variation for the inter-quartile area as well as for the outliers compared to the savanna class. Moreover the barren class shows the highest $A_{P}$-value (around 0.4 ) and the lowest $\psi$ value (around $30^{\circ}$ ).

a)

b)
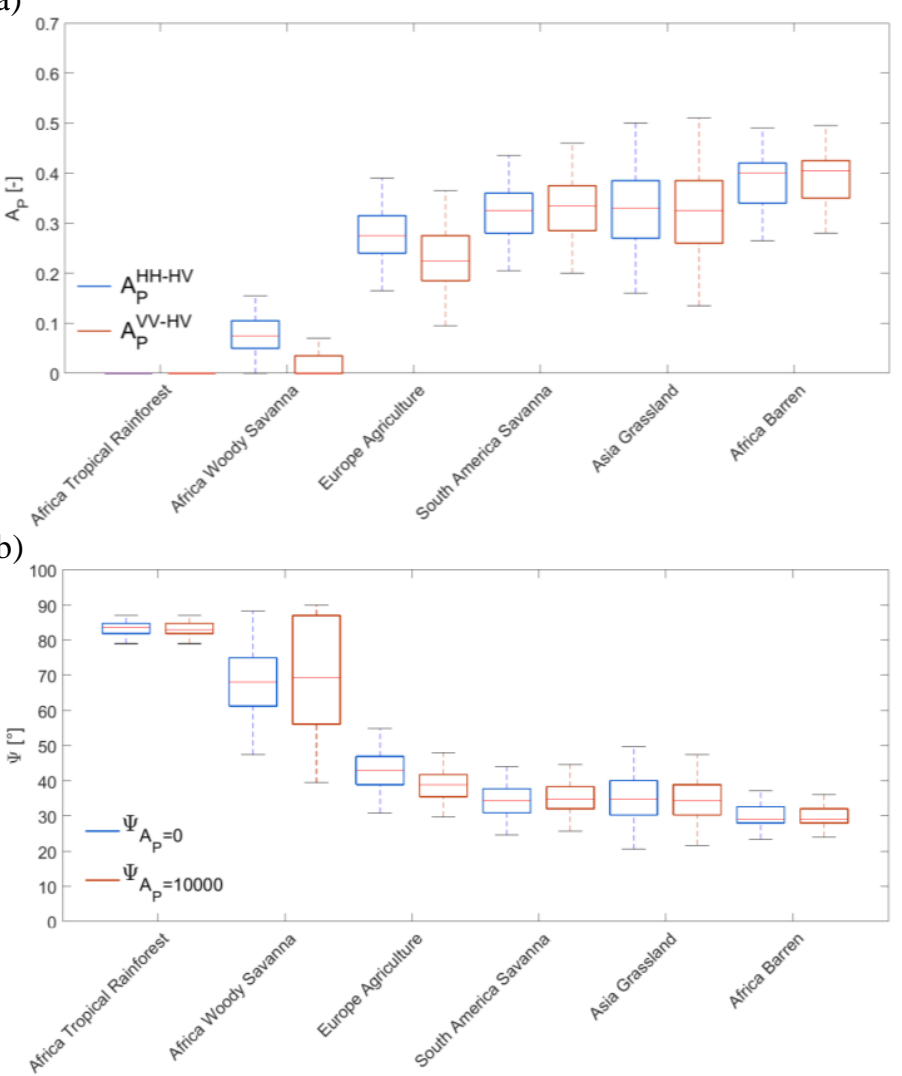

Fig. 10. Box plots of retrieved time-averaged vegetation shape (particle anisotropy) $A_{P}[-]$ (a) and vegetation orientation distribution width $\psi\left[{ }^{\circ}\right]$ (b) for selected target areas: tropical rain forest (Africa), light green: woody savanna (Africa), red: agriculture (Europe), green: savanna (South America), orange: grassland (Asia), dark red: barren land (Africa); The red horizontal line within each box indicates the median value; The box represents the interquartile region $(25 \%-75 \%)$ and the whiskers show the extent to the minimum and maximum value of $A_{P}$ and $\psi$, respectively.

\section{Comparison of Retrieved Vegetation Structure Parameters with Vegetation Height \& Above Ground Biomass Datasets}

A direct validation with in situ data for vegetation shape and main orientation $\left(A_{P}\right.$ and $\left.\psi\right)$ is intricate due to unavailability of appropriate measurements representing these model-based parameter estimates and due to the scale gap between in situ measurements (meter scale) of monitoring networks and remote sensing estimates (kilometer scale).

Hence, instead of validation with in situ measurements, which might be more or less of different type and therefore rather not comparable, an alternative strategy is suggested by globally comparing $A_{P^{-}}$and $\psi$-estimates with independent remotesensing based products of above ground biomass and vegetation height. Both independently retrieved products are explained in detail in [51] and [52].

It is anticipated that with increasing biomass and height of vegetation the orientation distribution proceeds towards $90^{\circ}$ 
indicating a random distribution of the vegetation elements due to increase in complexity of vegetation structure. Fig. 11 confirms this anticipated trend for both vegetation parameters (biomass \& height). Beyond $150 \mathrm{Mg} / \mathrm{ha}$ and $30 \mathrm{~m}$ height, the estimated vegetation orientation distribution ranges between

a)

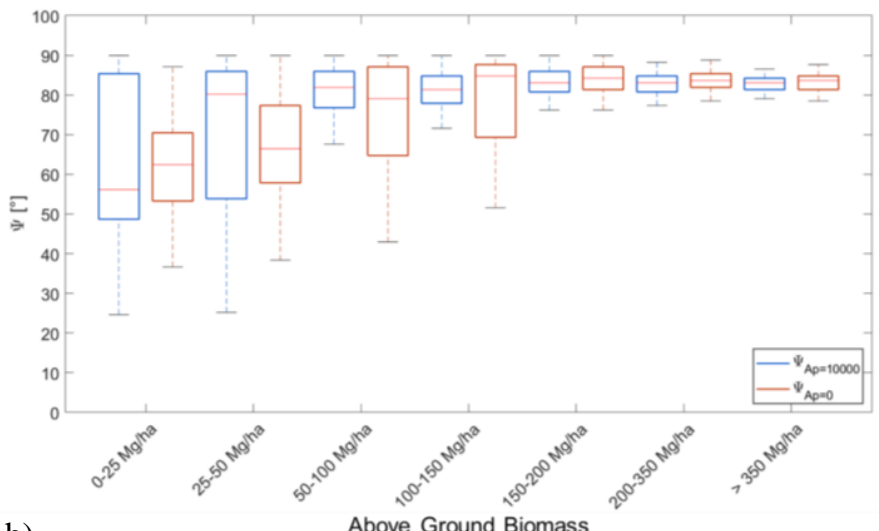

b)

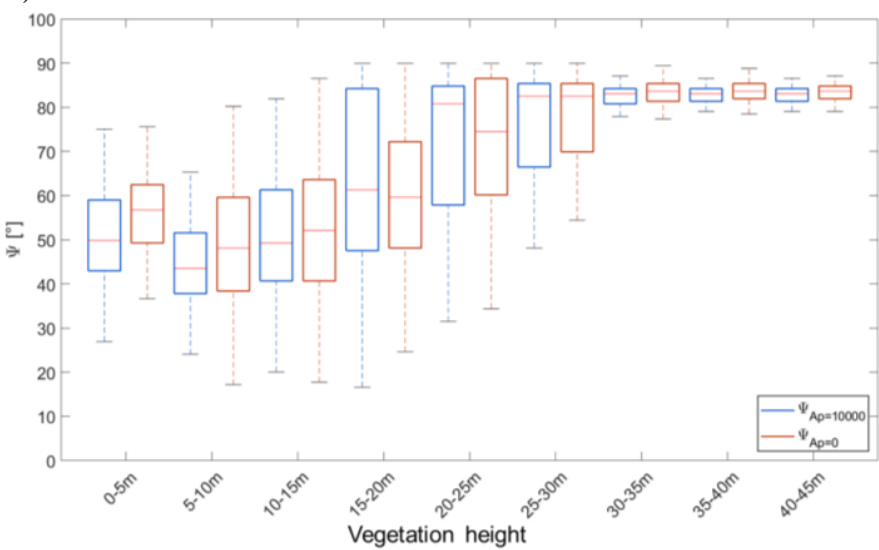

Fig. 11. Box plots of retrieved time-averaged vegetation orientation distribution width $(\psi)\left[^{\circ}\right]$ where red color indicates the assumption of vertical dipoles $\left(A_{P}=0\right)$ and blue color the assumption of horizontal dipoles $\left(A_{P}=\right.$ $10000)$; Box plots are displayed for different levels of above ground biomass [Mg/ha] [52] (a) and of vegetation height [m] [51] (b); The red horizontal line within each box indicates the median value; The box represents the interquartile region $(25 \%-75 \%)$ and the whiskers show the extent to the minimum and maximum value of $\psi$.

$80^{\circ}$ and $90^{\circ}$ which indicates a large variety of plant element orientations in the vegetation canopy structure. Both $\psi-$ estimates $\left(A_{P}=0 \& A_{P}=10000\right)$ follow similar trends along vegetation biomass and height, but with differing intra-class variance especially for medium to low vegetation cover. It is interesting that the lowest class $(0-5 \mathrm{~m})$ of vegetation height seems to be significantly biased by ground scattering influences (spatial and vertical heterogeneity of canopy) and shows higher values of $\psi$ than following (larger) vegetation height classes. This might be partly explained by the spaceborne LiDAR-based derivation methodology of the vegetation height dataset explained in [51].

Figure 12 presents the shape of the vegetation elements, estimated from the SMAP L-band radar data, versus biomass and height of vegetation from independent datasets. It is expected that with increasing vegetation cover (biomass \& height) the estimated elements of the canopy tend to a dipole- like shape $\left(A_{P}=0\right)$. For biomass beyond $150-200 \mathrm{Mg} / \mathrm{ha}$ and heights above $30 \mathrm{~m}$ this hypothesis is fulfilled. However, it has to be stated that even for medium to low vegetation biomass and height the absolute values for $A_{P}$ stay on a low level of 0.1 to 0.3 , whereas the range of physically possible values extends until 1.0 (spheres). Moreover, $A_{P}$-estimates of both polarimetric combinations (HH-HV \& VV-HV) show similar trends, but different intra-class variance. This is more pronounced for low to medium vegetation cover (biomass < $50 \mathrm{Mg} / \mathrm{ha}$ and height $<20 \mathrm{~m}$ ).

Figures $11 \& 12$ confirm by comparison with two independent vegetation datasets (above ground biomass \& height) that estimates of orientation distribution and shape of vegetation elements are consistent with measurable vegetation properties and have a physical interpretation.

a)
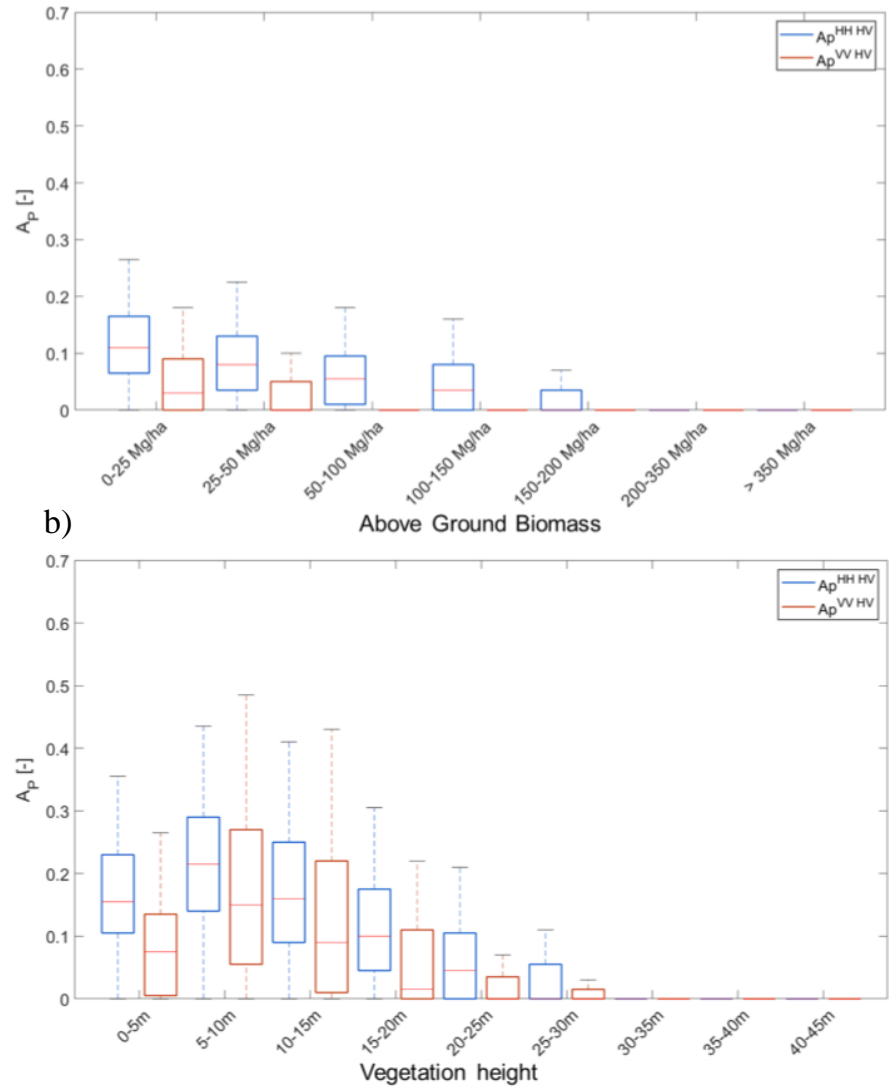

Fig. 12. Box plots of retrieved time-averaged vegetation shape (particle anisotropy) $\left(A_{P}\right)[-]$ where red color indicates the derivation from $\mu_{V V-H V}$ and blue color the derivation from $\mu_{H H-H V}$; Box plots are displayed for different levels of above ground biomass [Mg/ha] (a) and of vegetation height [m] (b); The red horizontal line within each box indicates the median value; The box represents the interquartile region $(25 \%-75 \%)$ and the whiskers show the extent to the minimum and maximum value of $A_{P}$.

This is in line with Figs. 6, 8 and 10 showing the distribution of vegetation structure parameters $\left(A_{P} \& \psi\right)$ along (selected) IGBP land cover classes. However, direct measurements of these model-based vegetation structure parameters are parsimonious to non-existent rendering a direct in situ validation as very challenging. 


\section{Discussion}

The results in Section IV show the possibility to estimate model-based vegetation structure parameters $\left(A_{P}, \psi\right)$ from three incoherent (no phase information), multi-polarimetric $\left(\left|S_{H H}\right|^{2},\left|S_{H V}\right|^{2},\left|S_{V V}\right|^{2}\right)$ SMAP L-band radar observations. Numerous satellite missions can provide these measurements or have them archived (e.g. SMAP, ALOS-2, SAOCOM \& AQUARIUS). Hence, the applicability can be broadened beyond SMAP to these other space-borne radar sensors as well as their planned successors like NISAR [58], Rose-L [59] or Tandem-L [60].

However, the assumptions of having one layer of homogeneously filled spheroids as a vegetation model exhibiting only single scattering and no multiple scattering should be sufficient for L-band (and lower frequencies) application, but not for C-band and higher frequencies [61]. This limits the application to low microwave frequency sensors.

Moreover, the penetration into the vegetation canopy decreases with increasing frequency [62]. In order to sense the entire above-ground vegetation canopy, longer wavelengths like L-band are preferred. Nonetheless, the results indicate so far that local spatial heterogeneity can be used to separate the ground and vegetation scattering component within the mixed (soil-vegetation) signal for a vegetation-only retrieval of the structure parameters $\left(A_{P}, \psi\right)$. This is subject to a potential limitation for sparse vegetation cover where first results in [45], [54] and [63] indicate additional influences from the soil conditions on the radar signal. However, a dedicated study is needed to clarify these additional interfering influences in the future.

In addition, it has to be mentioned that the spatial resolution of the radar data and the extend of the neighboring area, which was used to estimate the local spatial heterogeneity, will reflect into the retrieval results for $A_{P}$ and $\psi$. Hence, a scale transfer to higher or lower spatial resolution for structure representation might not be possible.

It is important to understand that strongly oriented vegetation estimates with $\psi$-values lower than $20^{\circ}$ do not occur for any land cover on the globe (see Fig. 6). This does not mean that this aligned vegetation type, e.g. stalk-dominated crops like barley or wheat, do not exist. It is rather the case that the coarse spatial resolution of the SMAP radar sensor $(9 \mathrm{~km}$, aggregated from $3 \mathrm{~km}$ ) in contrast to the sparse appearance and small spatial extent of strongly aligned vegetation within a kilometer-wide resolution cell leads to an underrepresentation. Hence, it would be interesting to apply the approach to higher-resolution SAR sensors, like ALOS-2, SAOCOM or NISAR, in a future study to see the difference in retrieved structure parameters and to understand if strongly aligned vegetation structures can be found at higher spatial resolution.

When focusing on the retrieved $A_{P}$-values, it is unexpected that $A_{P}$-values do not reach higher than 0.6 , which is still significantly different from spherical shapes $\left(A_{P}=1\right)$. However, Fig. $2 \mathrm{~b}$ indicates that the corresponding $\mu_{P P-P Q}$-values fed into the $A_{P}$-retrieval need to have values of 20 and higher to obtain these kind of $A_{P}-$ values. Figures $4 \mathrm{a}$ and $4 \mathrm{~b}$ indicate that such high $\mu_{P P-P Q^{-}}$values are not present in the global $\mu_{P P-P Q^{-}}$ data from SMAP for vegetated soil regions.

In addition, the retrieved vegetation structure parameters $\left(A_{P}\right.$, $\psi$ ) need to be understood in the light of a methodological limitation: The retrieval method is a two-step process, whereby the orientation distribution width $\psi$ is estimated first with pre-defined $A_{P}$-values (vertical dipoles: $A_{P}=0$, horizontal dipoles: $\left.A_{P}=10000\right)$. This has the advantage that dependencies of $\mu_{H H-H V}^{M o d e l}$ and $\mu_{V V-H V}^{M o d e l}$ on the orientation angle distribution width $\psi$ are equivalent, when either vertical $\left(A_{p}=0\right)$ or horizontal $\left(A_{p}=10000\right)$ dipoles are assumed for respective particle shape (see Fig. 2a). Moreover from Fig. 2a, both dipole selections $\left(A_{p}=0 \& A_{p}=10000\right)$ are needed to obtain the full information on the orientation angle distribution width $\psi$ for one polarization combination.

The particle anisotropy $A_{P}$ is retrieved assuming a fixed $\psi-$ value of $90^{\circ}$. With this assumption, $\mu_{H H-H V}^{M o d e l}$ and $\mu_{V V-H V}^{M o d e l}$ have the same dependencies within an $A_{p}$-range from zero (fully oriented dipoles) to one (spheres) (see Fig. 2b). It is recommended to extend the study at hand for the development of a single step approach under the support of fully polarimetric observations. Here, Bayesian methods may help to investigate the full parameter space.

Moreover, it stays challenging to directly validate these model-based, space-borne radar resolution-scale vegetation structure parameters $\left(A_{P} \& \psi\right)$, as a fully equivalent in situ measurement is non-existent, especially not at the size of satellite footprints. But sophisticated vegetation characterization is urgently needed for remote sensing signal decomposition to assess the conditions on ground below vegetation cover.

Nevertheless, the retrieved vegetation structure parameters have practical implications on application of model-based polarimetric decompositions like in [17], [30], [39]. Here $A_{P}$ and $\psi$ can be used as adequate input parameters for the discrete scatterer model representing the vegetation. This should facilitate polarimetric decompositions of ground and vegetation scattering components and improve ground parameter retrieval, like for soil moisture and surface roughness [37], [38].

Szigarski et al. [56] analyzed the radar vegetation index (RVI) using forward simulations with the discrete particle scattering model of (1). They also calculated the RVI from global SMAP L-band radar data. They show that the classical RVI of [56] needs to be corrected for soil scattering contributions at longer wavelength, e.g. L-band. They suggest a multi-senor-based correction in [56]. The retrieved vegetation structure parameters in this study can be directly applied in the forward model formulas for RVI, presented in [56], to calculate an improved (vegetation-only) radar vegetation index for global vegetation cover analyses. This might be especially helpful for longer wavelength sensing (L- \& P-band) where soil scattering contributions are more likely included in the recorded signals. The retrieved vegetation structure parameters might be also helpful to parameterize the vegetation attenuation in passive 
microwave (radiometer) radiative transfer approaches using the classical model of Mo et al., 1982 [64]. Here $A_{P}$ and $\psi$ can contribute to the simulation of the vegetation optical depth (VOD) using models, e.g. from Jackson \& Schmugge, 1991 [65].

Moreover, environmental Earth System models might benefit from first-order estimations of shape and orientation distribution of main vegetation elements from the presented retrieval [66]. This fulfills the purpose to inform globally and at large spatial scales rather than locally with (tree- or standbased) in situ observations.

\section{CONCLUSIONS AND OUTLOOK}

In this research study, we presented a radar-based estimation approach for two model-based vegetation structure parameters (shape $A_{P}$ and orientation distribution $\psi$ of main canopy elements). The approach is based on a small observation set of three incoherent (no phase information) polarimetric intensities $\left(\left|S_{H H}\right|^{2},\left|S_{H V}\right|^{2},\left|S_{V V}\right|^{2}\right)$ combined with a twoparameter $\left(A_{P} \& \psi\right)$ discrete scatterer model. The objective was to understand if this confined set of information can be sufficient to estimate these vegetation structure parameters from L-band signals.

Hence, the sub-pixel spatial heterogeneity, expressed by the covariation of co- and cross-polarized backscatter $\Gamma_{P P-P Q}$ of the neighboring cells, is taken to be indicative for the amount of vegetation-only co-to-cross-polarized backscatter ratio $\mu_{P P-P Q}$ moving out soil scattering influences and allowing a vegetation-only retrieval of vegetation shape $A_{P}$ and orientation distribution $\psi$.

However, the retrievals of the two parameters are not possible simultaneously, but consecutively, while a pre-assumption on either $A_{P}$ or $\psi$ has to be made. Hence, the retrievals are not independent, but show adequate estimates for the different land covers and global spatial distributions. For instance, tropical forests indicate randomly oriented dipoles as predominant vegetation structure type which is already indicated in [17] and [67]. The focus study on six different regions of interest, spanning from barren land to tropical rainforest, shows a steady increase of orientation distribution towards randomly oriented volumes and a continuous decrease in shape arriving at dipoles for tropical vegetation. For the barren regions, e.g. Sahara, the highest $A_{P}$-value (around 0.4) and the lowest $\psi$-value (around $30^{\circ}$ ) are obtained.

A comparison with independently derived datasets of vegetation height and above ground biomass confirms the consistent and meaningful retrieval of $A_{P}$ and $\psi$. However, it is a challenge to directly validate the model-based, spaceborne radar resolution-scale vegetation structure estimates $\left(A_{P}\right.$ $\& \psi)$, as a fully equivalent in situ measurement is nonexistent, especially not at the size of satellite footprints.

Nonetheless, vegetation monitoring from space benefits from the proposed approach as also multi-polarimetric data (no phase information) can add value to assess vegetation structural parameters. In the light of upcoming space-borne active microwave missions (e.g. L- and S-band NISAR \& Pband BIOMASS [68]) the technique could be applied right away to characterize vegetation canopies on global and regional scales at S-, L- and P-band frequencies. This is especially relevant for NISAR, which has a significantly higher spatial resolution (in the order of meters) than the SMAP radar instrument (in the order of kilometers).

Moreover, the retrieved vegetation structure parameters could be directly applied for the vegetation scattering component of model-based polarimetric decompositions. This should facilitate decomposition into ground and vegetation scattering components and improve retrieval of soil parameters (moisture $\&$ roughness) under vegetation.

\section{REFERENCES}

[1] Barrett, B.W. and G.P. Petropoulos. 2013. Satellite Remote Sensing of Surface Soil Moisture. In: G. P. Petropoulos, editor Remote Sensing of Land Surface Turbulent Fluxes And Soil Moisture: State of the Art. Taylor \& Francis Book. p. 85-120.

[2] Huang, Y., J.P. Walker, Y. Gao, X. Wu and A. Monerris. 2016. Estimation of Vegetation Water Content From the Radar Vegetation Index at L-Band. IEEE Transactions on Geoscience and Remote Sensing 54: 981-989.

[3] Kim, Y., T. Jackson, R. Bindlish, S. Hong, G. Jung and K. Lee. 2014. Retrieval of Wheat Growth Parameters With Radar Vegetation Indices. IEEE Geoscience and Remote Sensing Letters 11: 808-812.

[4] Sinha, S., C. Jeganathan, L.K. Sharma and M.S. Nathawat. 2015. A review of radar remote sensing for biomass estimation. Int J Environ Sci Te 12: 1779-1792.

[5] Vereecken, H., L. Weihermüller, F. Jonard and C. Montzka. 2012. Characterization of Crop Canopies and Water Stress Related Phenomena using Microwave Remote Sensing Methods: A Review. Vadose Zone Journal 11.

[6] van Emmerik, T., S.C. Steele-Dunne, J. Judge and N. van de Giesen. 2015. Impact of Diurnal Variation in Vegetation Water Content on Radar Backscatter From Maize During Water Stress. IEEE Transactions on Geoscience and Remote Sensing 53: 3855-3869.

[7] McNairn, H. and J. Shang. 2016. A Review of Multitemporal Synthetic Aperture Radar (SAR) for Crop Monitoring. In: Y. Ban, editor Multitemporal Remote Sensing: Methods and Applications. Springer International Publishing, Cham. p. 317-340.

[8] McDowell, N.G., N.C. Coops, P.S.A. Beck, J.Q. Chambers, C. Gangodagamage, J.A. Hicke, et al. 2015. Global satellite monitoring of climate-induced vegetation disturbances. Trends in Plant Science 20: 114-123.

[9] Chauhan, N.S., R.H. Lang, and K.J. Ranson. 1991. Radar modelling of a boreal forest. IEEE Transactions on Geoscience and Remote Sensing 29: 627-37.

[10] Attema, E.P.W. and F.T. Ulaby. 1978. Vegetation Modeled as a Water Cloud. Radio Science 13: 357-364.

[11] Graham, A.J. and R. Harris. 2003. Extracting biophysical parameters from remotely sensed radar data: a review of the water cloud model. Prog Phys Geog 27: 217-229.

[12] Paris, J.F. 1986. The Effect of Leaf Size on the Microwave Backscattering by Corn. Remote Sensing of Environment 19: 81-95.

[13] Van Leeuwen, H.J.C. and J.G.P.W. Clevers. 1994. Synergy between optical and microwave remote sensing for crop growth monitoring. Proceedings of the sixth symposium of physical measurements and signatures in remote sensing. Val d'Isere, France, 17-21 January. Toulouse: Centre Nationale d'Etudes Spatiales, 1175-82.

[14] Kweon, S.K. and Y. Oh. 2015. A Modified Water-Cloud Model With Leaf Angle Parameters for Microwave Backscattering From Agricultural Fields. IEEE Transactions on Geoscience and Remote Sensing 53: 28022809.

[15] Ulaby, F.T., K. Sarabandi, K. Mcdonald, M. Whitt and M.C. Dobson. 1990. Michigan Microwave Canopy Scattering Model. International Journal of Remote Sensing 11: 1223-1253.

[16] Ulaby, F.T., R.K. Moore and A.K. Fung. 1986. Microwave Remote Sensing: Active and Passive, From Theory to Applications, Vol. 3Artech House, Delham, Mass, USA.

[17] Freeman, A. and S.L. Durden. 1998. A three-component scattering model for polarimetric SAR data. IEEE Trans. on Geosci.Remote Sens. 36: 963-973. 
[18] Cloude, S. and E. Pottier. 1997. An entropy based classification scheme for land applications of polarimetric SAR. IEEE Trans. Geosci. Remote Sens. 35(1): 68-78.

[19] Steele-Dunne, S.C., H. McNairn, A. Monsivais-Huertero, J. Judge, P.W. Liu and K. Papathanassiou. 2017. Radar Remote Sensing of Agricultural Canopies: A Review. IEEE Journal of Selected Topics in Applied Earth Observations and Remote Sensing 10: 2249-2273.

[20] McNairn, H., J. Shang, X. Jiao, and B. Deschamps. 2012. Establishing crop productivity using RADARSAT-2. Int. Arch. Photogramm. Remote Sens. Spat. Inf. Sci. 39-B8: 283-287.

[21] Wiseman, G., H. McNairn, S. Homayouni, and J. Shang. 2014. RADARSAT-2 polarimetric SAR response to crop biomass for agricultural production monitoring. IEEE J. Sel. Topics Appl. Earth Observ. Remote Sens. 7 (11): 4461-4471.

[22] Zwieback, S. and I. Hajnsek. 2016. Influence of Vegetation Growth on the Polarimetric Zero-Baseline DInSAR Phase Diversity; Implications for Deformation Studies. IEEE Transactions on Geoscience and Remote Sensing 54: 3070-3082.

[23] Reigber, A. and A. Moreira. 2000. First demonstration of airborne SAR tomography using multibaseline L-band data. Ieee Transactions on Geoscience and Remote Sensing 38: 2142-2152.

[24] Lombardini, F. 2005. Differential tomography: A new framework for SAR interferometry. Ieee Transactions on Geoscience and Remote Sensing 43: 37-44.

[25] Minh, D.H.T., T. Le Toan, F. Rocca, S. Tebaldini, L. Villard, M. RejouMechain, et al. 2016. SAR tomography for the retrieval of forest biomass and height: Cross-validation at two tropical forest sites in French Guiana. Remote Sensing of Environment 175: 138-147.

[26] Huang, Y., J. Levy-Vehel, L. Ferro-Famil and A. Reigber. 2017. ThreeDimensional Imaging of Objects Concealed Below a Forest Canopy Using SAR Tomography at L-Band and Wavelet-Based Sparse Estimation. Ieee Geoscience and Remote Sensing Letters 14: 1454-1458.

[27] Chakraborty, M., K.R. Manjunath, S. Panigrahy, N. Kundu and J.S. Parihar. 2005. Rice crop parameter retrieval using multi-temporal, multiincidence angle Radarsat SAR data. Isprs Journal of Photogrammetry and Remote Sensing 59: 310-322.

[28] Santi, E., S. Paloscia, S. Pettinato, G. Fontanelli, M. Mura, C. Zolli, et al. 2017. The potential of multifrequency SAR images for estimating forest biomass in Mediterranean areas. Remote Sensing of Environment 200: 63-73.

[29] Canisius, F., J. Shang, J. Liu, X. Huang, B. Ma, X. Jiao, et al. 2017. Tracking crop phenological development using multi-temporal polarimetric Radarsat-2 data. Remote Sensing of Environment 210: 508518.

[30] Cloude, S.R. 2010. Polarisation: Applications in Remote Sensing, Oxford, Oxford University Press, U.K..

[31] Neumann, M., L. Ferro-Famil and A. Reigber. 2010. Estimation of Forest Structure, Ground, and Canopy Layer Characteristics From Multibaseline Polarimetric Interferometric SAR Data. IEEE Transactions on Geoscience and Remote Sensing 48: 1086-1104.

[32] Capodici, F., A. Maltese, G. Ciraolo, G. D'Urso and G. La Loggia. 2017. Power Sensitivity Analysis of Multi-Frequency, Multi-Polarized, MultiTemporal SAR Data for Soil-Vegetation System Variables Characterization. Remote Sensing 9(7): 677.

[33] $\mathrm{Du}$, L.J. and W.H. Peake. 1969. Rayleigh scattering from leaves, Proceedings of the IEEE 57(6): 1227-1229.

[34] Karam, M.A. and A.K. Fung. 1981. Scattering from randomly oriented circular disc with application to vegetation. Radio Science 18: 557-565.

[35] Lang, R.H. 1983. Electromagnetic backscattering from a sparse distribution of lossy dielectric scatterers. Radio Science: 16: 15-30.

[36] Lang, R.H. and J.S. Sidhu. 1983. Electromagnetic Backscattering From a Layer of Vegetation: A Discrete Approach. IEEE Transactions on Geoscience and Remote Sensing, GE-21: 62-71.

[37] Jagdhuber, T. 2012. Soil Parameter Retrieval under Vegetation Cover Using SAR Polarimetry, PhD Thesis, University Potsdam, Potsdam, published online under: http://nbn-resolving.de/urn:nbn:de:kobv:517opus-60519.

[38] Jagdhuber, T., I. Hajnsek and K.P. Papathanassiou. 2014. An Iterative Generalized Hybrid Decomposition for Soil Moisture Retrieval under Vegetation Cover Using Fully Polarimetric SAR, IEEE Journal of Selected Topics in Applied Earth Observations and Remote Sensing 8 (8): 3911-3922.

[39] Lee, J.S. and E. Pottier. 2009. Polarimetric Radar Imaging: From Basics to Applications. Boca Raton, USA, CRC Press.
[40] Jagdhuber, T., D. Entekhabi, A.G. Konings, K.A. McColl, S.H. Alemohammad, N.N. Das, C. Montzka and M. Piles. 2016. Physicallybased retrieval of SMAP active-passive measurements covariation and vegetation structure parameters. In: Proceedings of the 2016 IEEE International Symposium on Geoscience and Remote Sensing, 10-15 July, Beijing, China, 3078-3081.

[41] Entekhabi, D., E.G. Njoku, P.E. O'Neill, K.H. Kellogg, W.T. Crow, W.N. Edelstein, J.K. Entin, S.D. Goodman, T.J. Jackson, J. Johnson, J. Kim-ball, J.R. Piepmeier, R.D. Koster, N. Martin, K.C. McDonald, M. Moghaddam, S. Moran, R. Reichle, J.-C. Shi, M.W. Spencer, S.W. Thurman, L. Tsang and J.J. Van Zyl. 2010. The Soil Moisture Active Passive (SMAP) Mission. Proceedings of the IEEE 98: 704-716.

[42] Entekhabi, D., N.N. Das, E.G. Njoku, J.T. Johnson, and J. Shi. 2016. SMAP L2 Radar/Radiometer Half-Orbit 9 km EASE-Grid Soil Moisture, Version 3. Boulder, Colorado USA. NASA National Snow and Ice Data Center Distributed Active Archive Center. doi: https://doi.org/10.5067/8YTYSV6JGBK2.

[43] Chan, S. 2013. Ancillary Data Report: Static Water Fraction. Jet Propuls. Lab. Calif. Inst. Technol., JPL, D- 53059.

[44] Das, N.N. 2013. Ancillary Data Report: Urban Areas. Jet Propuls. Lab. Calif. Inst. Technol., JPL, D-53060.

[45] Das, N.N., D. Entekhabi, E.G. Njoku, J.J.C. Shi, J.T. Johnson and A Colliander. 2014. Tests of the SMAP Combined Radar and Radiometer Algorithm Using Airborne Field Campaign Observations and Simulated Data, IEEE Transactions on Geoscience and Remote Sensing 52 (4): 2018-2028.

[46] Entekhabi, D., N. Das, E. Njoku, J. Johnson and J. Shi. 2012. Soil Moisture Active Passive: Algorithm Theoretical Basis Document L2 \& L3 Radar/Radiometer Soil Moisture (Active/Passive) Data Products, JPL Report.

[47] Derksen, C., X. Xu, S.R. Dunbar, A. Colliander, Y. Kim, J.S. Kimball, T.A. Black, E. Euskirchen, A. Langlois, M.M. Loranty and P. Marsh. 2017. Retrieving landscape freeze/thaw state from Soil Moisture Active Passive (SMAP) radar and radiometer measurements. Remote Sensing of Environment 194: 48-62.

[48] Piepmeier J.R. et al. 2017. SMAP L-band microwave radiometer: Instrument design and first year on orbit, IEEE Trans. Geosci. Remote Sens. 55 (4): 1954-1966.

[49] Entekhabi D. et al. 2014. SMAP handbook, Jet Propuls. Lab., Pasadena, CA, USA, JPL Rep. 400-1567: 1-192. [Online]. Available: http://smap.jpl.nasa.gov/mission/description/

[50] West, R. et al. 2016. SMAP L1C Radar Half-orbit 3 km EASE-grid Backscatter, Version 3, SMAP_L1C_S0_HiRes_V3, Fairbanks, Alaska USA. Alaska Satellite Facility, http://dx.doi.org/10.5067/NVVUJ0MNG3PN.

[51] Simard, M., N. Pinto, J.B. Fisher, and A. Baccini. 2011. Mapping forest canopy height globally with spaceborne lidar. Journal of Geophysical Research: Biogeosciences 116 (G4): 1-12.

[52] Santoro, M., O. Cartus, S. Mermoz, A. Bouvet, T. Le Toan, N. Carvalhais, D. Rozendaal, M. Herold, V. Avitabile, S. Quegan, J. Carreiras, Y. Rauste, H. Balzter, C. Schmullius, F.M. Seifert. 2018. GlobBiomass global above-ground biomass and growing stock volume datasets, available on-line at http://globbiomass.org/products/globalmapping.

[53] Leroux, D. J., N.N. Das, D. Entekhabi, A. Colliander, E.G. Njoku, R.S. Dunbar, and S.J. Yueh. 2016. Active-passive disaggregation of brightness temperatures during the SMAPVEX12 campaign. IEEE Transactions on Geoscience and Remote Sensing. 54: 6859-6867.

[54] Wu, X., J.P. Walker, N.N. Das, R. Panciera, and C. Rüdiger. 2014. Evaluation of the SMAP brightness temperature downscaling algorithm using active-passive microwave observations. Remote sensing of environment. 155: 210-221.

[55] Wu, X., J.P. Walker, C. Rüdiger, and R. Panciera. 2015. Effect of landcover type on the SMAP active/passive soil moisture downscaling algorithm performance. IEEE Geoscience and remote sensing letters. 12: 846-850.

[56] Szigarski, C., T. Jagdhuber, M. Baur, C. Thiel, M. Parrens, J.P. Wigneron, M. Piles and D. Entekhabi. 2018. Analysis of the Radar Vegetation Index and Potential Improvements. Remote Sensing 10(11): 1776.

[57] Kim, Y. and J.J. van Zyl. 2000. On the Relationship between Polarimetric Parameters. In: Proceedings of the 2000 IEEE International Symposium on Geoscience and Remote Sensing, Honolulu, USA, 24-28 July: $1298-1300$. 
[58] Rosen, P., et al. 2017. The NASA-ISRO SAR (NISAR) mission dualband radar instrument preliminary design. In: Proceedings of the 2017 IEEE International Symposium on Geoscience and Remote Sensing, Fort Worth, USA, 23-28 July: 3832-3835.

[59] Pierdicca, N., et al. 2019. The Copernicus L-band SAR mission ROSE-L (Radar Observing System for Europe)(Conference Presentation). In: Active and Passive Microwave Remote Sensing for Environmental Monitoring III. International Society for Optics and Photonics, 9-12 September, Strasbourg, France: 111540E.

[60] Moreira, A., G. Krieger, I. Hajnsek, K.P. Papathanassiou, M. Younis, P. Lopez-Dekker, S. Huber, M. Villano, M. Pardini, M. Eineder, F. De Zan, and A. Parizzi. 2015. TandemL: a highly innovative bistatic SAR Mission for global observation of dynamic processes on the Earth's surface. IEEE Geoscience and Remote Sensing Magazine 3: 8-23.

[61] Picard, G., T. Le Toan, and F. Mattia. 2003. Understanding C-band radar backscatter from wheat canopy using a multiple-scattering coherent model. IEEE Transactions on Geoscience and Remote Sensing 41(7): 1583-1591.

[62] Baur, M., T. Jagdhuber, M. Link, M. Piles, R. Akbar, and D. Entekhabi. 2018. Multi-Frequency Estimation of Canopy Penetration Depths from SMAP/AMSR2 Radiometer and IceSAT Lidar Data. In: Proceedings of the 2018 IEEE International Symposium on Geoscience and Remote Sensing, 22-27 July, Valencia, Spain: 365-368.

[63] Montzka, C., T. Jagdhuber, R. Horn, H.R. Bogena, I. Hajnsek, A. Reigber, and H. Vereecken. 2016. Investigation of SMAP fusion algorithms with airborne active and passive L-band microwave remote sensing. IEEE Transactions on Geoscience and Remote Sensing. 54: 3878-3889.

[64] Mo, T., B.J. Choudhury, T.J. Schmugge, J.R. Wang and T.J. Jackson. 1982. A Model for Microwave Emission From Vegetation-Covered Fields. J. Geophys. Res 87: 11229-11237.

[65] Jackson, T.J. and T.J. Schmugge. 1991. Vegetation effects on the microwave emission of soils. Remote Sens. Environ. 36: 203-212.

[66] Arora, V., 2002. Modeling vegetation as a dynamic component in soilvegetation-atmosphere transfer schemes and hydrological models. Reviews of Geophysics 40(2): 1-26.

[67] Freeman, A., 2007. Fitting a two-component scattering model to polarimetric SAR data from forests. IEEE Transactions on Geoscience and Remote Sensing 45(8): 2583-2592.

[68] Le Toan, T., Quegan, S., Davidson, M.W.J., Balzter, H., Paillou, P., Papathanassiou, K., Plummer, S., Rocca, F., Saatchi, S., Shugart, H. and Ulander, L. 2011. The BIOMASS mission: Mapping global forest biomass to better understand the terrestrial carbon cycle. Remote sensing of environment, 115(11), pp.2850-2860. 